\title{
A study on boundary-layer transition induced by free-stream turbulence
}

\author{
A. C. MANDAL ${ }^{1}$, L. VENKATAKRISHNAN ${ }^{2}$ AND J.DEY D \\ ${ }^{1}$ Department of Aerospace Engineering, Indian Institute of Science, Bangalore 560012, India \\ ${ }^{2}$ EAD, National Aerospace Laboratories, Bangalore 560017, India
}

(Received 6 November 2008; revised 29 April 2010; accepted 29 April 2010; first published online 15 July 2010)

\begin{abstract}
Boundary-layer transition at different free-stream turbulence levels has been investigated using the particle-image velocimetry technique. The measurements show organized positive and negative fluctuations of the streamwise fluctuating velocity component, which resemble the forward and backward jet-like structures reported in the direct numerical simulation of bypass transition. These fluctuations are associated with unsteady streaky structures. Large inclined high shear-layer regions are also observed and the organized negative fluctuations are found to appear consistently with these inclined shear layers, along with highly inflectional instantaneous streamwise velocity profiles. These inflectional velocity profiles are similar to those in the ribboninduced boundary-layer transition. An oscillating-inclined shear layer appears to be the turbulent spot-precursor. The measurements also enabled to compare the actual turbulent spot in bypass transition with the simulated one. A proper orthogonal decomposition analysis of the fluctuating velocity field is carried out. The dominant flow structures of the organized positive and negative fluctuations are captured by the first few eigenfunction modes carrying most of the fluctuating energy. The similarity in the dominant eigenfunctions at different Reynolds numbers suggests that the flow prevails its structural identity even in intermittent flows. This analysis also indicates the possibility of the existence of a spatio-temporal symmetry associated with a travelling wave in the flow.
\end{abstract}

Key words: boundary layers, transition to turbulence

\section{Introduction}

In the absence of any unique route to transition, the laminar-turbulent transition process continues to interest all. In a low free-stream turbulence environment, this process in a laminar boundary layer begins with the growth of the TollmeinSchlichting wave, before eventual breakdown to turbulence with the appearance of spikes (in the hot-wire signal) in the high shear-layer region (e.g. Klebanoff, Tidstrom \& Sargent 1962; Nishioka, Asai \& Iida 1981). The slow viscous TollmeinSchlichting wave mechanism may be bypassed at an elevated level of free-stream turbulence (Morkovin 1969), and the rapid transition process that occurs is widely accepted as the bypass transition. Because of its importance in practical flows, bypass transition has extensively been studied in the past (e.g. Dryden 1936; Taylor

$\dagger$ Email address for correspondence: jd@aero.iisc.ernet.in 
1939; Arnal \& Juillen 1978; Leventhal \& Reshotko 1981; Kendall 1985, 1990; Blair 1992; Westin et al. 1994). Kendall (1998) reviewed some of these experimental studies. Dryden (1936) and Taylor (1939) observed very low frequency streamwise velocity fluctuations of large amplitude in the boundary layer. The Dryden-Taylor observation did not receive much attention until Klebanoff (1971) demonstrated that disturbances with narrow spanwise spread grow more or less linearly with the boundary-layer thickness. Klebanoff (1971) referred to these disturbances as the 'breathing modes', because, as noted earlier by Taylor (1939), they appeared to correspond to a thickening and thinning of the boundary layer. Kendall (1991) called them the Klebanoff modes and this name seems to have taken hold enough even though they are not modes in the sense of being eigenfunctions.

Kendall $(1985,1990)$, in his receptivity studies, finds that the low-frequency fluctuation is due to the streaky structure originating from the leading edge. Boundarylayer perturbation due to such streaky structures, though random in time and space, is not turbulence. From carefully made measurements in pre-transitional boundary layers on a flat plate, Westin et al. (1994) find that $u_{r \operatorname{ms}, \max }$ grows linearly with the square root of the distance from the leading edge, as also observed by Klebanoff (1971); $u$ denotes the fluctuating streamwise velocity component. The proportionality constants differ from experiment to experiment even when the amplitudes were normalized by the free-stream turbulence level. Matsubara \& Alfredsson (2001) find from their flow visualization and hot-wire measurements that free-stream disturbances induce streamwise streaks of high and low streamwise velocities in the boundary layer. In the downstream direction, the spanwise wavelength of streaks is of the order of the boundary-layer thickness. They also find that $u_{r m s, \max }^{2}$, i.e. the energy of the streamwise velocity fluctuation, grows linearly with the downstream distance, for $\gamma \leqslant 20 \%$, where $\gamma$ is the flow intermittency. Fransson, Matsubara \& Alfredsson (2005), who studied bypass transition with focus on modelling the transition zone, find that the transition Reynolds number is inversely proportional to $T u^{2}$ (where $T u$ denotes the turbulence intensity).

While the appearance of streamwise streaks in bypass transition has been established by various investigators, the direct numerical simulation (DNS) of bypass transition by Jacobs \& Durbin (2001), who compared their results with the carefully made measurements of Roach \& Brierly (1992), shows a certain pattern of the streamwise fluctuating velocity component, apart from longitudinal streaks. The organized positive and negative $u$ fluctuations are designated by these authors as forward jets and backward jets, respectively. They also suggest that backward jets contain the low-speed fluid lifted from the wall region, while forward jets, which occur near the wall, contain high-speed fluid. In the spanwise plane, they find an asymmetric pattern between a narrow region with intense negative $u$ and a broad region with weak positive $u$. The simulation by Brandt, Schlatter \& Henningson (2004) also shows streamwise-elongated regions of high and low streamwise velocities. For steady streaks in a stable boundary layer, the algebraic growth theory (e.g. Butler \& Farrell 1992; Andersson, Berggren \& Henningson 1999; Luchini 2000) provides adequate description. According to this theory, optimal disturbances create streamwise counterrotating vortices, which in turn can generate elongated streamwise streaks by the lift-up effect. Matsubara \& Alfredsson (2001) have found that the initial growth of the disturbance and its wall-normal shape are in accordance with the optimal perturbation theory of Luchini (2000). On the other hand, Zaki \& Durbin (2005) suggest that the interaction between two low-frequency continuous Orr-Sommerfeld modes or one high-frequency and one low-frequency mode can also generate streaks. 
The former interaction cannot trigger transition and decays downstream, whereas the latter one triggers transition.

Flow visualization studies of actual bypass transition reveal that unsteady streaks are wavy before they break down to turbulence (e.g. Matsubara \& Alfredsson 2001; Mans et al. 2005), and these wavy streaks are often found to be of sinuous or varicose mode. Jacobs \& Durbin (2001) find no evidence of sinuous, or other prefatory streak instability and suggest that spot precursors are localized instability of a single jet. Furthermore, they suggest that turbulent spots are not created by forward jets, though very intense with a magnitude of as high as $25 \%$ of the free-stream velocity, but by the interaction of long backward jets of the fluctuating streamwise velocity with free-stream eddies at the boundary-layer edge. In their DNS, Brandt et al. (2004), however, find sinuous-like and varicose-like breakdowns, which are driven by the spanwise shear and the wall-normal shear, respectively. They also report that the varicose-like breakdown shows similarity with the breakdown scenario of Jacobs \& Durbin (2001). Furthermore, they suggest that the interaction between low-speed and high-speed streaks plays an important role in the formation of an incipient spot.

Although some studies (e.g. Jacobs \& Durbin 2001; Durbin \& Wu 2007) proposed the Kelvin-Helmholtz-type instability arising from backward jets as the spot precursor, the possibility of the secondary instability of streaks cannot be ignored and has led many investigators (e.g. Andersson et al. 2001; Asai, Minagawa \& Nishioka 2002; Brandt \& Henningson 2002) to study the aspect of secondary instability in a controlled manner, both experimentally and theoretically. Asai et al. (2002) performed controlled experiments by exciting only the symmetric varicose or the anti-symmetric sinuous mode in the boundary layer. They find the anti-symmetric mode of low-speed streak to be more unstable than the symmetric mode when the streak width is of the order of the shear-layer thickness. Elofsson, Kawakami \& Alfredsson (1999) applied wall suction to generate streaky structures in a plane Poiseuille flow in studying the characteristics of the secondary instability on these streaks with and without forcing; using two earphones, both symmetric (varicose) and antisymmetric (sinuous) modes were forced. From the controlled forcing of the secondary instability, they find the sinuous mode to be the dominating one than the varicose mode. Andersson et al. (2001) have shown that the sinuous spanwise oscillation of the low-speed region is the most dangerous secondary instability. They also find that the streak critical amplitude beyond which travelling waves are excited is $26 \%$ of the freestream velocity. Brandt \& Henningson (2002) studied the late stages of transition originating from the sinuous scenario of Andersson et al. (2001). They observed elongated quasi-streamwise vortices which are located on the flanks of the low-speed streak. Recently, Mans, de Lange \& van Steenhoven (2007) have investigated bypass transition in a water channel using the particle-image velocimetry (PIV)-laser induced fluorescence (LIF) technique and find evidence of the sinuous mode. We may note that their measurements were restricted to the spanwise plane only.

Past experimental studies of bypass transition have revealed many aspects of this complex process, as mentioned above. However, there are many aspects such as the structure of the fluctuating velocities, specially in the wall-normal plane, and incipient turbulent spots which are generally unsteady and occur over a spatial extent (Jacobs \& Durbin 2001), and are yet to be experimentally investigated in details in an actual bypass transition; moreover, smoke flow visualizations (e.g. Matsubara \& Alfredsson 2001) and the PIV-LIF technique of Mans et al. (2007) were confined to the spanwise plane. The PIV technique, in contrast to a single-point measurement technique, can capture the instantaneous flow field simultaneously in the entire measurement 
zone (e.g. Adrian, Meinhart \& Tomkins 2000b). This study is aimed at studying various flow structures in the wall-normal and spanwise planes in bypass transition using PIV, with an emphasis on the wall-normal plane.

Since PIV provides a large number of instantaneous data over a large spatial extent, proper orthogonal decomposition (POD) of these data may be useful in extracting the dominant flow structures. POD is applied to decompose a random function into a weighted linear sum of orthogonal eigenfunctions (see Holmes, Lumley \& Berkooz 1996 for details). The first $n$ eigenfunctions modes of POD can capture more energy on an average than the first $n$ functions of any other basis. The orthogonality of the POD eigenfunctions can be utilized in constructing a reduced-order model with these eigenfunctions as the basis functions. Since its first application in fluid mechanics by Lumley (1967), POD has found wide applications in many flow problems (e.g. Aubry et al. 1988; Rajaee, Karlsson \& Sirovich 1994; Rempfer \& Fasel 1994; Gunes \& Rist 2004; Hasan \& Sanghi 2007). Rempfer \& Fasel (1994) exploited the idea of spacetime symmetry to extract the lambda structures in a developing transitional boundary layer. Gunes \& Rist (2004) performed POD analysis of their simulated controlled and uncontrolled transitional boundary layer in order to reconstruct and control transition. They found that the most energetic POD modes for uncontrolled and controlled modes show a striking similarity and most of the flow energy is captured by a few POD modes. Rajaee et al. (1994), who experimentally studied a forced transitional mixing layer, found that a low-order system of dynamical equations obtained by projecting the Navier-Stokes equations on the basis set of empirical eigenfunction modes predicts the short-time dynamics adequately. Another aspect of the present investigation is to extract the dominant POD eigenfunction modes of bypass transition.

The paper is organized as follows. The experimental details, measurement techniques are described in $\S 2$, and the POD methodology is described in $\S 3$. The experimental results in the wall-normal and spanwise planes are presented in $\S 4$. The results of the POD study are presented in $\S 5$, followed by a discussion in $\S 6$. The results are summarized in $\S 7$.

\section{Experimental details}

\subsection{Wind tunnel}

The experiments were carried out in a low-turbulence wind tunnel, the details of which are reported in Vasudevan, Dey \& Prabhu (2001) and Banerjee, Mandal \& Dey (2006). Briefly, this was an open circuit tunnel with a square test section of dimensions $500 \mathrm{~mm} \times 500 \mathrm{~mm} \times 3000 \mathrm{~mm}$. The settling chamber ahead of the contraction had a smooth entry section with honeycomb and screens $(4$ screens; $8 \mathrm{mesh} / \mathrm{cm})$. The contraction ratio was $14: 1$, and its shape ensured a monotonic velocity increase. The test section had a divergence of about $1 / 487$ over $3 \mathrm{~m}$ on both side walls to correct for the side-wall boundary-layer growth. The tunnel had a short diffuser which was isolated from the rest of the tunnel by a flexible rubber band to minimize the transmission of vibrations to the test section. The tunnel speed was controlled by a speed controller that regulated a $5 \mathrm{~kW}$ DC motor, which drove the fan at the diffuser end. The maximum tunnel speed is about $22 \mathrm{~m} \mathrm{~s}^{-1}$. The free-stream streamwise turbulence intensity was $0.1 \%$ and both the cross-flow turbulent intensities were $0.05 \%$ at $15 \mathrm{~m} \mathrm{~s}^{-1}$, as measured using a cross-wire probe in two different planes. The signals from the cross-wire probe were amplified using an $\mathrm{AC}$ gain and then filtered using a $3 \mathrm{~Hz}$ high pass filter. The cross-wire was calibrated following Simon, Qiu \& 
Yuan (2000) and the fluctuating velocities were estimated following Perry (1982). The boundary-layer measurements were made on a flat plate that was placed horizontally in the mid-plane of the test section. The plate leading edge was of super-ellipse shape (Narasimha \& Prasad 1994), which merged smoothly with the plate; this shape had been used in many earlier constant pressure experiments (Narasimha et al. 1984; Vasudevan et al. 2001; Banerjee et al. 2006). Since the plate was made of aluminium, the reflection of the laser sheet from the plate was not desirable as it contaminated the measurements. A smooth black sticker of $0.1 \mathrm{~mm}$ thickness was stuck to the plate covering the leading edge to reduce such reflection (Banerjee et al. 2006). Both the hot-wire and the PIV measurements reported here were made on this smooth black surface.

\subsection{Particle image velocimetry}

The PIV unit (IDTpiv, USA) consisted of a double-cavity Nd:YAG laser (New Wave Research, $100 \mathrm{~mJ}$ ), laser sheet optics, a CCD camera (Sharpvision 1400DE of $1360 \times 1036$ pixel resolution) and the associated data processing software (proVISION II). The CCD camera was equipped with a $50 \mathrm{~mm}$ focal length lens (Sigma). The camera could capture five image pairs per second. The software was based on a meshfree second-order accurate algorithm capable of providing the velocity information at closely spaced points, simultaneously. A pair of single exposed images was subdivided into subimages (Interrogation window) for statistical correlation and the spatial shift of particles was quantified for the velocity information. A Gaussian interpolation procedure was applied to achieve a subpixel resolution. A masking method was enabled in this package to eliminate the errors due to the finite interrogation window size, e.g. loss of pairs and image truncation. To account for the velocity and seed density gradient effects that generally occur in practical PIV recordings, the processing software had a high-resolution feature (Lourenco \& Krothapalli 2000; Krothapalli et al. 2003); a detailed discussion of the processing technique and comparison with standard PIV algorithms are available in Lourenco \& Krothapalli (2000).

Figure 1 shows a schematic diagram of the PIV set-up. The camera was placed perpendicular to the laser sheet that illuminated the wall-normal $(x-y)$ plane. A similar arrangement was made for the spanwise $(x-z)$ plane measurements. The streamwise distance from the plate leading edge is denoted by $x$, the wall-normal distance is denoted by $y$, and $z$ is the spanwise distance. The measurement area is denoted by $\Delta x \times \Delta y$ in the $x-y$ plane, and in the $x-z$ plane by $\Delta x \times \Delta z$. The measurements in the $x-y$ plane were made along the plate centreline, $z=0$. The PIV measurement zones were $\Delta x \times \Delta y \approx 50 \mathrm{~mm} \times 40 \mathrm{~mm}$ in the $x-y$ plane, and $\Delta x \times \Delta z \approx 57 \mathrm{~mm} \times 44 \mathrm{~mm}$ in the $x-z$ plane. The measurements were made with the camera centred at a distance $x_{1}=x+\Delta x / 2$ from the plate leading edge. The laser sheet optics was equipped with a spherical lens and a cylindrical lens. The spherical lens reduced the laser beam diameter and the cylindrical lens expanded it into a sheet. The laser sheet thickness was $2 \mathrm{~mm}$, which is comparable to the sheet thicknesses of $0.5 \mathrm{~mm}$ and $2 \mathrm{~mm}$ used by Adrian et al. (2000b) and Kostas, Soria \& Chong (2002), respectively. The flow was seeded with smoke particles $(\sim 1 \mu \mathrm{m}$; EUROLITE smoke fluid) generated by a commercial fog generator (HP Line), which was placed about $3 \mathrm{~m}$ ahead of the tunnel entrance. The smoke flow rate was controlled in such a way that there were enough particles during the measurements. Due to the less image intensity near the sides of the acquired images, we did not consider the sides of these images. Using an interrogation window size of $24 \times 24$ pixels, the acquired images were processed with a $100 \times 100$ rectangular mesh to obtain 10000 vectors per frame. The interpolation 


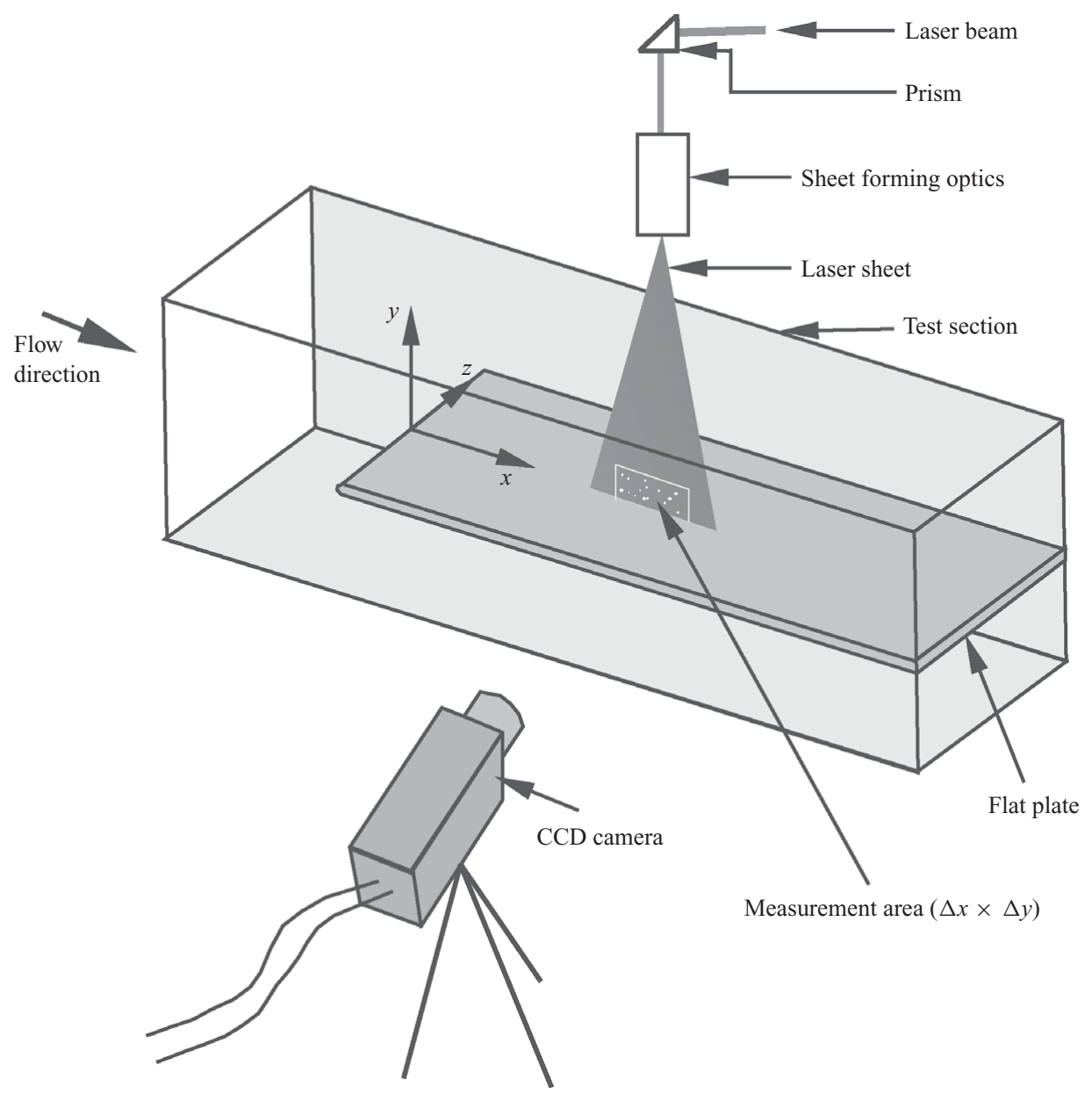

FIGURE 1. A schematic diagram of the PIV set-up in the wall-normal plane.

limit, which could be set in the processing software, was five vectors in 10000 vectors and frames with more than five interpolated vectors were thus skipped. An errorchecking option based on a multi-layer check algorithm associated with proVISION II was enabled to ensure that the vector was a valid one through a qualifier flag. Although a high spatial resolution feature is incorporated in the processing software, the spatial resolution of the measurements in terms of the correlation window size was approximately $0.9 \mathrm{~mm}$ in the wall-normal plane and $1 \mathrm{~mm}$ in the spanwise plane (i.e. $\delta x \times \delta y \approx 0.9 \mathrm{~mm} \times 0.9 \mathrm{~mm}$ and $\delta x \times \delta z \approx 1 \mathrm{~mm} \times 1 \mathrm{~mm}$ in the $x-y$ and $x-z$ planes, respectively). The experimental parameters, i.e. the field of view, correlation window, spatial resolution and laser sheet thickness in this study are comparable with those reported by other investigators (e.g. Liu, Adrian \& Hanratty 2001; Kostas et al. 2002; Pedersen \& Meyer 2002). The wall was identified by magnifying the image of a small pin $(0.7 \mathrm{~mm}$ diameter $)$ on the wall with an accuracy of $\pm 0.1 \mathrm{~mm}$. However, this accuracy in determining the wall may be a drawback with this measurement technique. The PIV measurements were validated by comparing with the standard Blasius and turbulent boundary-layer flows (Mandal 2005); the same PIV system was also used in our previous works (Banerjee et al. 2006; Mandal, Venkatakrishnan \& 


$\begin{array}{lccccccc}\text { Grid } & \text { Bar geometry } & M(\mathrm{~mm}) & d(\mathrm{~mm}) & \tau_{x}(u)(\mathrm{mm}) & \Lambda_{x}(u)(\mathrm{mm}) & x_{\text {grid }}(\mathrm{mm}) & T u(\%) \\ \text { Grid 1 } & \text { Circular } & 15 & 1.5 & 4( \pm 1) & 8.2 & -900 & 0.9 \\ \text { Grid 2 } & \text { Circular } & 25 & 2.5 & 5( \pm 1) & 11 & -1000 & 1.4 \\ \text { Grid 3 } & \text { Circular } & 50 & 5 & 6( \pm 1) & 14.6 & -950 & 2.8\end{array}$

TABLE 1. Grid details. The turbulent intensities are at the leading edge.
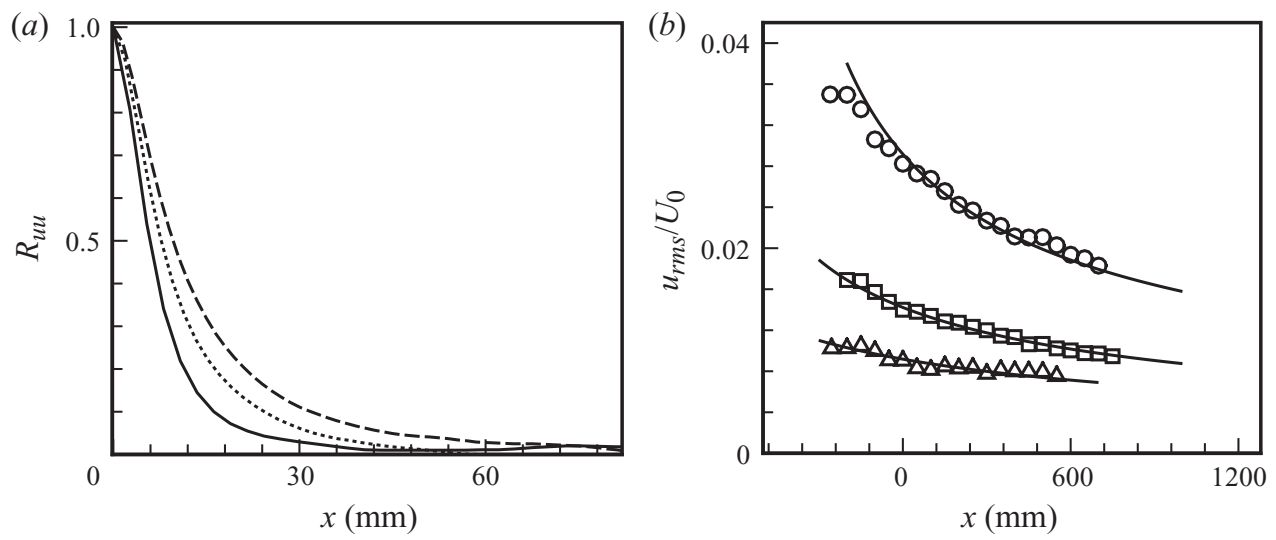

FIGURE 2. (a) Free-stream turbulence correlation coefficient at the leading edge. Lines:grid $1 ; \cdots \cdots$, grid $2 ;----$, grid 3. (b) Streamwise decay of $u_{r m s}$ in the free stream for three different grids. Symbols: $\triangle$, grid $1 ; \square$, grid $2 ; \bigcirc$, grid $3 ;-,(2.1)$.

Dey 2006). In the following, the mean velocity components are denoted by $\bar{U}, \bar{V}$ and $\bar{W}$, whereas the instantaneous velocity components are denoted by $U_{I}, V_{I}$ and $W_{I}$ in the $x-, y$ - and $z$-directions, respectively. The fluctuating velocity components are denoted by $u\left(=U_{I}-\bar{U}\right), v\left(=V_{I}-\bar{V}\right)$ and $w\left(=W_{I}-\bar{W}\right)$. The free-stream speed is $U_{0}$. The displacement thickness is denoted by $\delta^{*}$, and $\delta_{0}^{*}$ is the displacement thickness at $x_{1}$. The boundary-layer thickness based on $0.99 U_{0}$ at $x_{1}$ is denoted by $\delta_{0}$, and $H$ denotes the shape factor. The Reynolds number based on the displacement thickness is denoted by $R e_{\delta^{*}}$.

\subsection{Free-stream turbulence}

A constant-temperature hot-wire anemometer with $5 \mu \mathrm{m}$ tungsten wire and length-todiameter ratio of about 300 was used as the sensing element. The hot-wire data were acquired at $2 \mathrm{kHz}$, and no filter was used for the measurements of grid turbulence. In table 1 , the details of three different grids used to initiate transition are given; $M$ is the spacing between two bars, $d$ is the diameter of the bar, $\tau_{x}(u)$ is the Taylor microscale, $\Lambda_{x}(u)$ is the integral length scale and $x_{\text {grid }}(\mathrm{mm})$ is the distance of the grid from the leading edge. The Taylor microscale and the integral length scale of free-stream turbulence were estimated from the longitudinal correlation function, $R_{\text {ии }}(\boldsymbol{x})$, at the plate leading edge and shown in figure 2(a); this follows Matsubara \& Alfredsson (2001), who also estimated the Taylor microscale for the transverse velocity component from the correlation function. We may note that this spatial correlation was obtained from the auto-correlation function of $u$ and using the Taylor hypothesis. The estimated values of $\tau_{x}(u)$ and $\Lambda_{x}(u)$ are given in table 1 . The downstream decay of the free-stream turbulence intensity, $T u$, in figure $2(b)$ shows that the decay rate 


$\begin{array}{lcrc}\text { Grid } & C & x_{0} & b \\ \text { Grid 1 } & 0.62 & -1200 & -0.6 \\ \text { Grid 2 } & 0.78 & -800 & -0.6 \\ \text { Grid 3 } & 1.19 & -500 & -0.6\end{array}$

TABLE 2. Values of the constant, virtual origin and exponent for different grids.

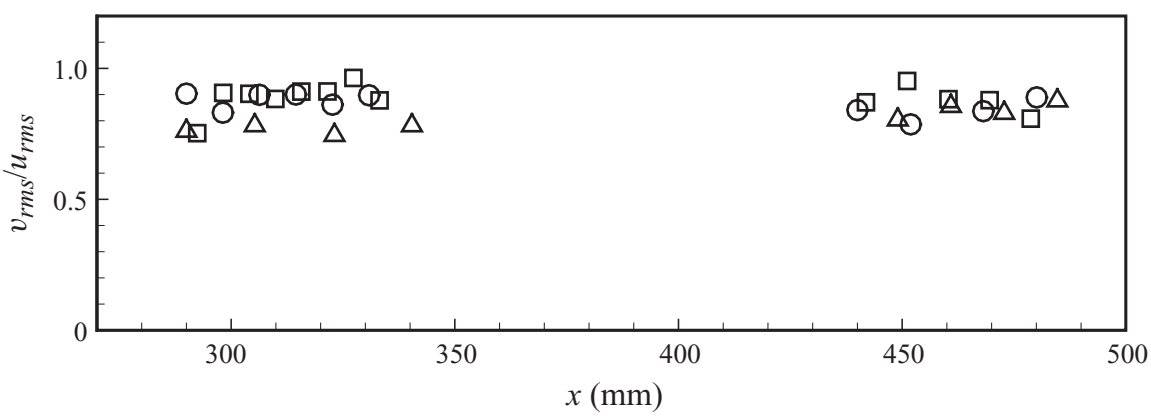

FiguRE 3. Values of $v_{r m s} / u_{r m s}$ at different locations in $\Delta x$ for different free-stream turbulence levels. Symbols: $\triangle$, grid $1 ; \square$, grid $2 ; \bigcirc$, grid 3 .

follows (Westin et al. 1994; Fransson et al. 2005)

$$
T u=u_{r m s} / U_{0}=C\left(x-x_{0}\right)^{b},
$$

where $x_{0}$ is the virtual origin, the exponent $b$ is the decay rate and $C$ is a constant for a particular grid. While the decay rate is expected to be $b=-0.5$ (Tennekes \& Lumley 1997), the present value of $b$ is -0.6 . This agrees well with those reported by Fransson et al. (2005) and Westin et al. (1994). The values of $C, x_{0}$ and $b$ for the grids used here are given in table 2.

The PIV measurements were made at two streamwise locations of $x_{1}=310$ and $460 \mathrm{~mm}$. With the camera centred at $x_{1}$, the streamwise extent covered by the PIV was $\Delta x \approx 50 \mathrm{~mm}$ in the $x-y$ plane. The values of $v_{r m s} / u_{r m s}$ in the free stream were measured at various points in $\Delta x \approx 50 \mathrm{~mm}$. Figure 3 shows the anisotropy measure in the downstream direction of the three different grids. For grids 2 and 3, the average value of $v_{r m s} / u_{r m s} \approx 0.9$ is consistent with the recent measurements by Fransson et al. (2005), who find this ratio to be $\geqslant 0.9$; for grid 1 , the value of 0.8 is slightly lower (see figure 3).

Figure 4 shows the streamwise variation of the free-stream pressure coefficient, $C_{p}=1-\left(U_{0} / U_{R}\right)^{2}$, in the presence of grids; here $U_{R}$ is the reference free-stream velocity. The initial strong favourable pressure gradient may be attributed to the influence of the leading edge. The $C_{p}$ variation in the region $150 \mathrm{~mm} \leqslant x \leqslant 700 \mathrm{~mm}$ is within $\pm 2 \%$. This is even less in the domain of our measurements. Similar variation in the pressure gradient was also reported in the measurements of Roach \& Brierly (1992) and Arnal \& Juillen (1978).

\section{POD methodology}

POD is used to find an orthogonal set of basis functions, $\boldsymbol{\Phi}(\boldsymbol{x})$, which can represent an ensemble of data optimally (Berkooz, Holmes \& Lumley 1993; Moreno et al. 


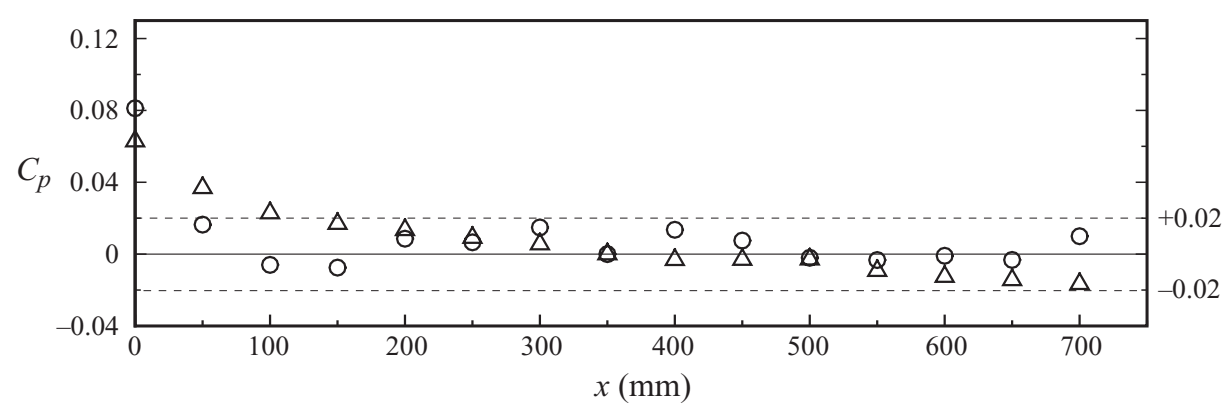

FIGURE 4. Streamwise variation of $C_{p}$ in the presence of grids. Symbols: $\triangle$, grid 1 ; $\bigcirc$, grid 3.

2004). That is, one needs to maximize the following inner product:

$$
\max _{\boldsymbol{\Phi}(\boldsymbol{x})} \frac{\left\langle|(\boldsymbol{v}(\boldsymbol{x}), \boldsymbol{\Phi}(\boldsymbol{x}))|^{2}\right\rangle}{\|\boldsymbol{\Phi}(\boldsymbol{x})\|^{2}}
$$

where $\boldsymbol{v}(\boldsymbol{x})$ is a random function such as the fluctuating velocity field in the present case; (,), \|\| and \langle\rangle denote the inner product in the Hilbert space of square intergrable functions, the corresponding norm and an averaging operation, respectively. A necessary condition for (3.1) to hold is that $\boldsymbol{\Phi}(\boldsymbol{x})$ needs to be the eigenfunctions of the integral eigenvalue equation (Berkooz et al. 1993):

$$
\int_{D} \boldsymbol{R}\left(\boldsymbol{x} ; \boldsymbol{x}^{\prime}\right) \boldsymbol{\Phi}\left(\boldsymbol{x}^{\prime}\right) \mathrm{d} \boldsymbol{x}^{\prime}=\lambda \boldsymbol{\Phi}(\boldsymbol{x}) .
$$

Here $D$ is the domain of integration, and $\boldsymbol{R}\left(\boldsymbol{x} ; \boldsymbol{x}^{\prime}\right)=\left\langle\boldsymbol{v}(\boldsymbol{x}) \boldsymbol{v}^{*}\left(\boldsymbol{x}^{\prime}\right)\right\rangle$ is a correlation function; the asterisk denotes a complex conjugate. The eigenfunctions can be normalized such that $\left(\boldsymbol{\Phi}^{k}, \boldsymbol{\Phi}^{l}\right)=\delta_{k l}$, where $\delta_{k l}$ denotes the Kronecker delta. The eigenfunction system $\boldsymbol{\Phi}^{k}$ is complete in the sense that the fluctuating velocity field, $\boldsymbol{v}(\boldsymbol{x}, t)$, can be expanded in terms of the orthogonal eigenfunctions:

$$
\boldsymbol{v}\left(\boldsymbol{x}, t_{n}\right)=\sum_{k} a^{k}\left(t_{n}\right) \boldsymbol{\Phi}^{k}(\boldsymbol{x})
$$

where

$$
a^{k}\left(t_{n}\right)=\left(\boldsymbol{v}\left(\boldsymbol{x}, t_{n}\right), \boldsymbol{\Phi}^{k}(\boldsymbol{x})\right) .
$$

The eigenfunctions were calculated here using the 'snapshot method' of Sirovich (1987). The discrete data of the fluctuating velocities from a PIV realization were arranged in vectors (Pedersen $\&$ Meyer 2002), $\hat{\boldsymbol{S}}_{i}$ :

$$
\hat{\boldsymbol{S}}_{i}=\boldsymbol{V}_{i}-\frac{1}{M} \sum_{j=1}^{M} \boldsymbol{V}_{j}, \quad i=1,2, \ldots, M,
$$

where $M$ is the number of ensemble and $\boldsymbol{V}_{i}$ is the instantaneous velocity. From these vectors, the elements of an covariance matrix are formed as

$$
R_{i j}=\left(\hat{S}_{i}, \hat{S}_{j}\right)
$$

which is an $M \times M$ matrix. Since the covariance matrix is symmetric, its eigenvalues, $\lambda_{i}$, are non-negative and its eigenvectors, $\phi_{i}(i=1,2, \ldots, M)$, form a complete orthogonal set. The total energy $E$ is defined as the sum of the eigenvalues of the covariance matrix, i.e. $E=\sum_{1}^{M} \lambda_{i}$, and the percentage of the relative energy carried by the $k$ th 


$\begin{array}{lcccccc}\text { Grid } & U_{0}\left(\mathrm{~m} \mathrm{~s}^{-1}\right) & \delta_{0}(\mathrm{~mm}) & \delta_{0}^{*}(\mathrm{~mm}) & H & R e_{\delta_{0}^{*}} & S_{n} \\ \text { Grid 1 } & 5.4 & 4.9 & 1.6 & 2.50 & 557 & 584 \\ \text { Grid 2 } & 4 & 6 & 2.09 & 2.46 & 539 & 591 \\ \text { Grid 3 } & 2.9 & 7.8 & 2.39 & 2.45 & 447 & 439\end{array}$

TABLE 3. Boundary-layer parameters in pre-transitional flows at $x_{1}=310 \mathrm{~mm}$.

\begin{tabular}{lcrccccc} 
Grid & $U_{0}\left(\mathrm{~m} \mathrm{~s}^{-1}\right)$ & $\gamma(\%)$ & $\delta_{0}(\mathrm{~mm})$ & $\delta_{0}^{*}(\mathrm{~mm})$ & $H$ & $R e_{\delta_{0}^{*}}$ & \multicolumn{1}{c}{$S_{n}$} \\
Grid 1 & 5.6 & 1 & 6.3 & 2.15 & 2.45 & 777 & 1823 \\
Grid 1 & 5.7 & 4 & 6.5 & 2.14 & 2.43 & 787 & 403 \\
Grid 1 & 6 & 25 & 7.2 & 2.10 & 2.25 & 813 & 541 \\
Grid 2 & 4.3 & 1 & 7.8 & 2.47 & 2.42 & 685 & 699 \\
Grid 3 & 3.2 & 1 & 8.5 & 2.68 & 2.41 & 553 & 1328 \\
Grid 3 & 3.4 & 4 & 8.8 & 2.58 & 2.24 & 566 & 1223 \\
Grid 3 & 3.6 & 20 & 9.0 & 2.46 & 2.21 & 571 & 1166
\end{tabular}

TABLE 4. Boundary-layer parameters in intermittent flows at $x_{1}=460 \mathrm{~mm}$.

mode is denoted by $E_{k}\left(=\left(\lambda_{k} / E\right) \times 100 \%\right)$. The orthogonal eigenfunctions are defined as

$$
\boldsymbol{\Phi}^{k}=\sum_{i=1}^{M} \phi_{i}^{k} \hat{\boldsymbol{S}}_{i}, \quad k=1, \ldots, M
$$

where $\phi_{i}^{k}$ is the $i$ th component of the $k$ th eigenvector (Kruse, Gunther \& Rohr 2003).

\section{Experimental results}

Three grids (in table 1) were used to create different free-stream turbulence levels. The PIV measurements of a pre-transitional and low intermittency flows in both the wall-normal and wall-parallel planes were carried out with the camera centred at $x_{1}=310$ and $460 \mathrm{~mm}$, respectively. Initially, a hot-wire was used to identify pretransitional and intermittent flows. The flow intermittency was changed by increasing the free-stream speed and keeping the hot-wire at $x_{1}=460 \mathrm{~mm}$. The flow intermittency, $\gamma$, was measured following the method adopted by Ramesh, Dey \& Prabhu (1996). Briefly, the velocity signal at $y / \delta_{0}^{*} \approx 1.3$ was sensitized by taking its double differential, and then squared. This made it easier to differentiate between the high- and lowfrequency components of the signal. A probability distribution of the squared signal was plotted, which was then used to identify a threshold. Above the threshold, the flow was considered to be turbulent, while it was non-turbulent below the threshold.

\subsection{Wall-normal plane}

The measured mean flow parameters in pre-transitional and intermittent flows are given in tables 3 and 4 , where $S_{n}$ is the number of PIV realizations. The mean velocities were obtained by averaging over the PIV realizations, $S_{n}$. In the following, the distances are normalized by $\delta_{0}^{*}$. The measured mean velocity profiles in the pretransitional and $\gamma \approx 1 \%$ flows are compared with the Blasius profile in figure $5(a)$. Compared to the Blasius profile, the measured mean velocity profiles show a gain in the inner part of the boundary layer and a deficit in the outer part, as is also reported by other investigators (Westin et al. 1994; Matsubara \& Alfredsson 2001). Figure $5(b)$ shows the $u_{r m s}$ distribution across the boundary layer, for grid 3 . The peak 

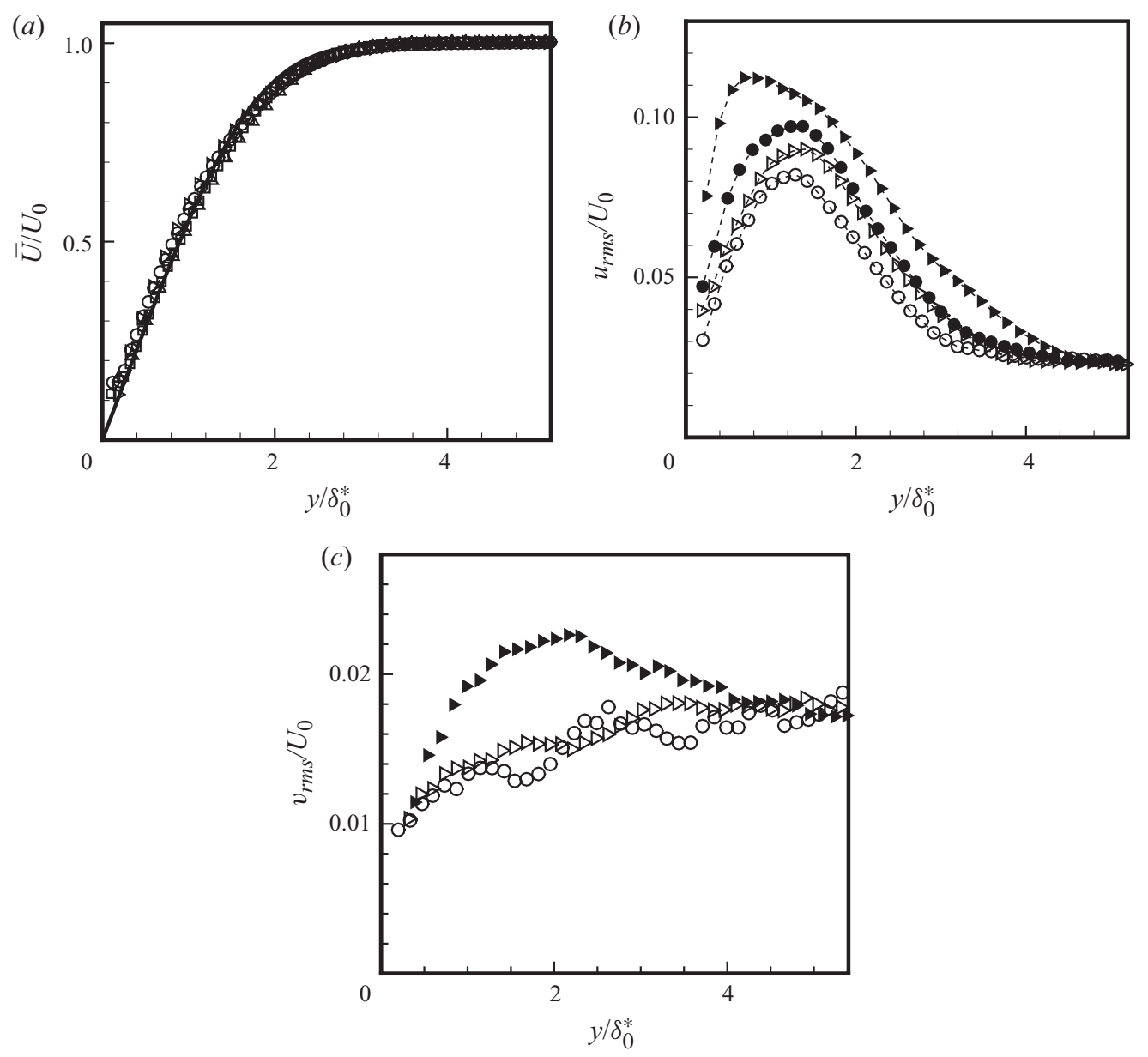

FIGURE 5. (a) Comparison of the measured mean velocity profiles in pre-transitional $\left(x_{1}=310 \mathrm{~mm}\right)$ and $\gamma \approx 1 \%\left(x_{1}=460 \mathrm{~mm}\right)$ flows with the Blasius profile. Symbols and lines: $\triangle, \gamma \approx 1 \%$ (grid 1 ); $\square, \gamma \approx 1 \%$ (grid 2); $\bigcirc$, pre-transition; $\triangleright, \gamma \approx 1 \%$ (grid 3 ); - , Blasius. (b) Measured $u_{r m s}$ profiles in pre-transitional and intermittent flows for grid 3. Symbols: - - ○ - -, pre-transition; - - - -, $\gamma \approx 1 \%$; - - - , $\gamma \approx 4 \%$ - - - -, $\gamma \approx 20 \%$. (c) Measured $v_{r m s}$ profiles in pre-transitional and intermittent flows for grid 3. Symbols: $\bigcirc$, pre-transition; $\triangleright, \gamma \approx 1 \% ; \gamma \approx 20 \%$.

$u_{r m s}$ increases with increasing intermittency and the peak is at about $y / \delta_{0}^{*} \approx 1.3$, for $\gamma \leqslant 4 \%$, while it moves towards the wall for the $\gamma \approx 20 \%$ flow. This is in agreement with other studies (e.g. Roach \& Brierly 1992; Jacobs \& Durbin 2001; Matsubara \& Alfredsson 2001). The typical distribution of $v_{r m s}$ shown in figure $5(c)$, for grid 3, is similar to those reported by others (e.g. Jacobs \& Durbin 2001; Inasawa et al. 2003) in the sense that the values are damped, compared to the free-stream values, while approaching towards the wall, for pre-transitional and $\gamma \approx 1 \%$ flows; in $\gamma \approx 20 \%$ flow, the growth is as expected.

A frame-by-frame examination of the entire PIV realizations revealed the following aspects. The boundary layer was seen to fluctuate, i.e. sometimes the boundary layer was thin and sometimes it was thick, as shown in the two instantaneous frames in figure 6, for pre-transitional flow with grid 3; the vectors in this figure are 

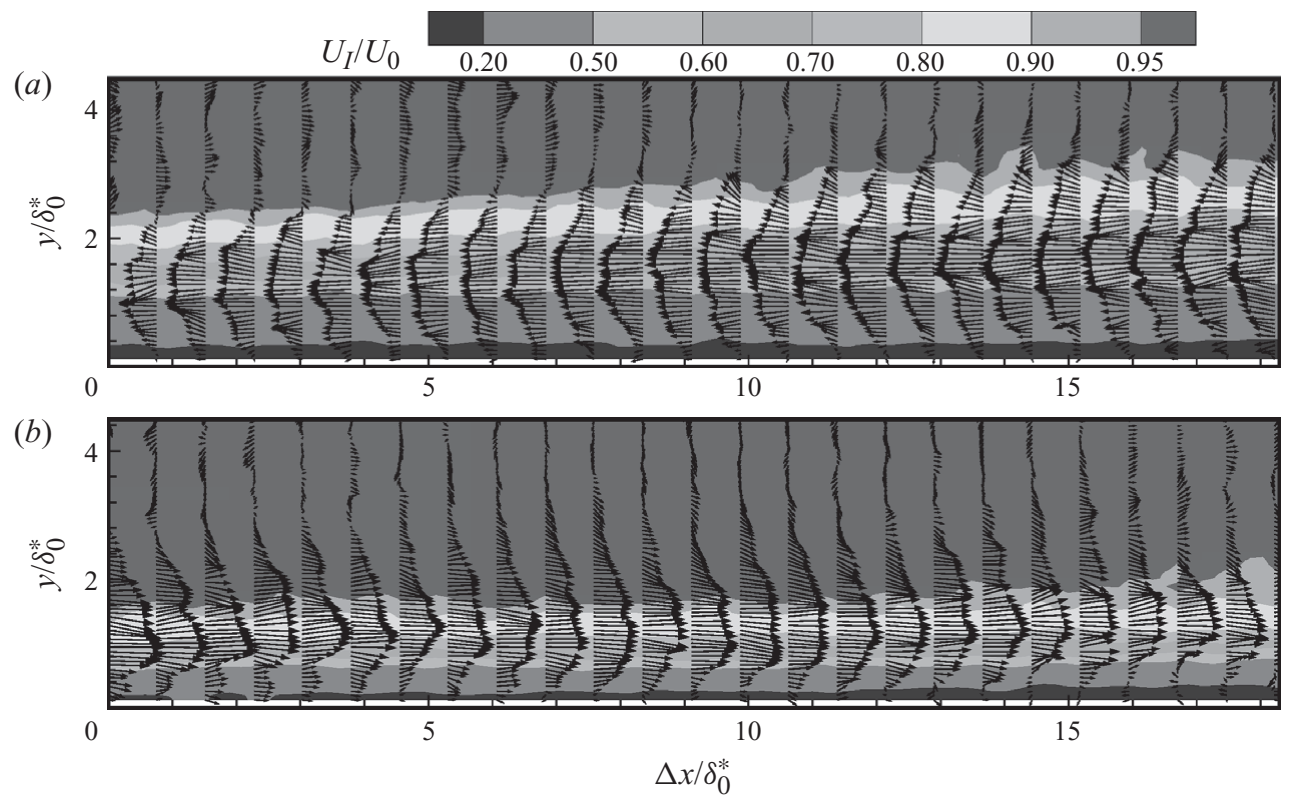

FIgURE 6. Two instantaneous PIV realizations in pre-transitional flow for grid 3; level indicates the value of $U_{I} / U_{0}$ and arrows are the fluctuating velocity vectors of $u$ and $v$. (a) Negative $u$ fluctuations and $(b)$ positive $u$ fluctuations.

the fluctuating velocity vectors over the contours of the instantaneous streamwise velocity. Such 'thinning' and 'thickening' of the boundary layer were also observed in intermittent flows. This 'breathing mode' flow feature was inferred by Klebanoff (1971) from the measured $u_{r m s}$ profiles. It is generally accepted that this 'breathing mode' is associated with low-frequency disturbances (Kendall 1991). As can be seen in this figure, the fluctuating velocity vectors appear as a jet-like structure with small $v$ and large $u$. Therefore, these fluctuations may be termed as negative $u$ fluctuations or positive $u$ fluctuations, depending on the sign. Jacobs \& Durbin (2001) and Zaki \& Durbin (2005) identify these structures as backward jets or forward jets. Backward jets do not mean that the total velocity $U_{I}(x, y, z, t)$ is reversed and it merely refers to the velocity relative to the local mean, i.e. $U_{I}(x, y, z, t)-\bar{U}(x, y)<0$; similarly, $U_{I}(x, y, z, t)-\bar{U}(x, y)>0$, for forward jets (Jacobs \& Durbin 2001). These negative and positive $u$ fluctuations may be attributed to unsteady streaks in the boundary layer, which manifest at an elevated level of free-stream turbulence (e.g. Matsubara \& Alfredsson 2001). Thus, a thick boundary layer with negative $u$ fluctuations is due to the low-speed streak and a thin boundary layer with positive $u$ fluctuations is due to the high-speed streak, as will also be evident in $\S 4.2$. The usual velocity decomposition, i.e. $u=U_{I}(x, y, z, t)-\bar{U}(x, y)$, for example, were used in various bypass transition studies (e.g. Alfredsson \& Matsubara 2000; Jacobs \& Durbin 2001; Inasawa et al. 2003) and was adopted here.

Figure 7 shows three typical instantaneous PIV realizations in the $\gamma \approx 1 \%$ flow, for grid 3. In this figure, the fluctuating velocity vectors are plotted over the contours of normalized instantaneous shear, $\left(\mathrm{d} U_{I} / \mathrm{d} y\right) \delta_{0} / U_{0}$. These frames mostly represent the flow under consideration. The first frame (figure $7 a$ ) shows a laminar-like flow as is also seen in several other PIV realizations. The small velocity fluctuation seen in the boundary layer is almost of the same order of the free-stream turbulence intensity. 

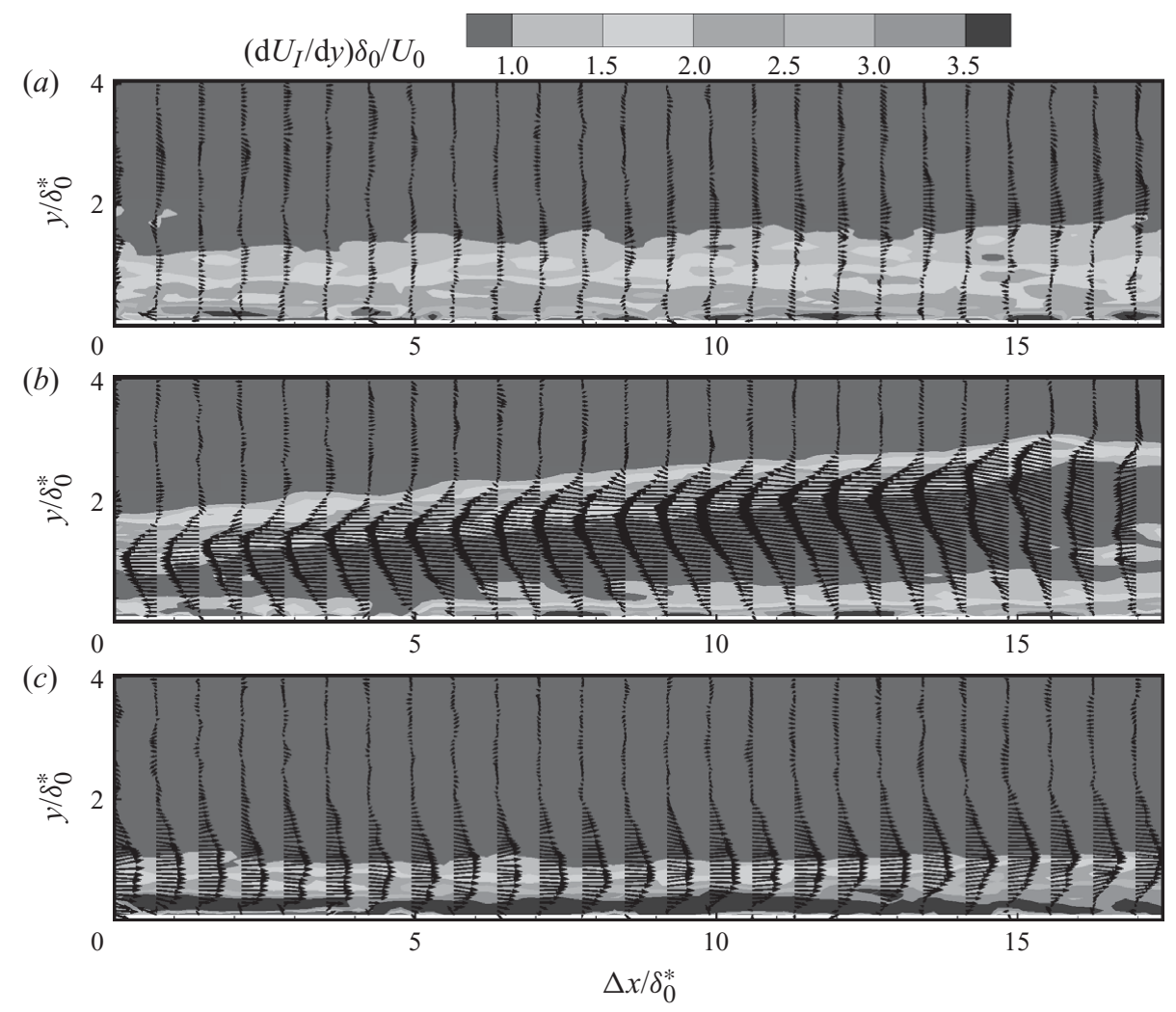

FIGURE 7. Fluctuating velocity vectors over the contours of normalized instantaneous shear, $\left(\mathrm{d} U_{I} / \mathrm{d} y\right) \delta_{0} / U_{0}$. (a) Laminar-like velocity field; $(b)$ negative $u$ fluctuations and the inclined shear layer; $(c)$ positive $u$ fluctuations and thinner shear layer near the wall. Grid $3, \gamma \approx 1 \%$ flow.

Figure 7(b) shows an inclined instantaneous shear layer with a structure of negative $u$ fluctuations. The main component of these fluctuations seems to be $u$, i.e. small $v$. We also find that the negative $u$ fluctuation is very strong towards the boundary-layer edge. The maximum value of $u / U_{0}$ is found to be approximately 0.33 . Figure $7(c)$ shows that a structure of positive $u$ fluctuations appears in a strong thin shear layer near the wall, compared to the shear layer in the laminar-like frame (figure 7a). Again, the main component of the fluctuations is $u$, and the maximum value of $u / U_{0}$ is found to be approximately 0.26 . Jacobs \& Durbin (2001) have reported similar structures of the fluctuating $u$-velocity field in their DNS of bypass transition. They also find the forward jet to be very intense and attains a value of $u / U_{0} \approx 0.25$. This compares very well with the present value of $u / U_{0} \approx 0.26$. Furthermore, their results also show that the forward jet is strong near the wall and a backward jet extends towards the boundary-layer edge, similar to that reported here. The present measurements thus experimentally confirm the jet-like structures of Jacobs \& Durbin (2001). We may note that similar $u$ fluctuations were also observed in other intermittent flows and the fluctuations in other pre-transitional flows studied were almost like those in figure 6. While the maximum value of $u / U_{0}$ was about 0.25 in pre-transitional flows, it was very often found to be more than $30 \%$ in an intermittent flow. This may be expected as the flow has already undergone breakdown. 

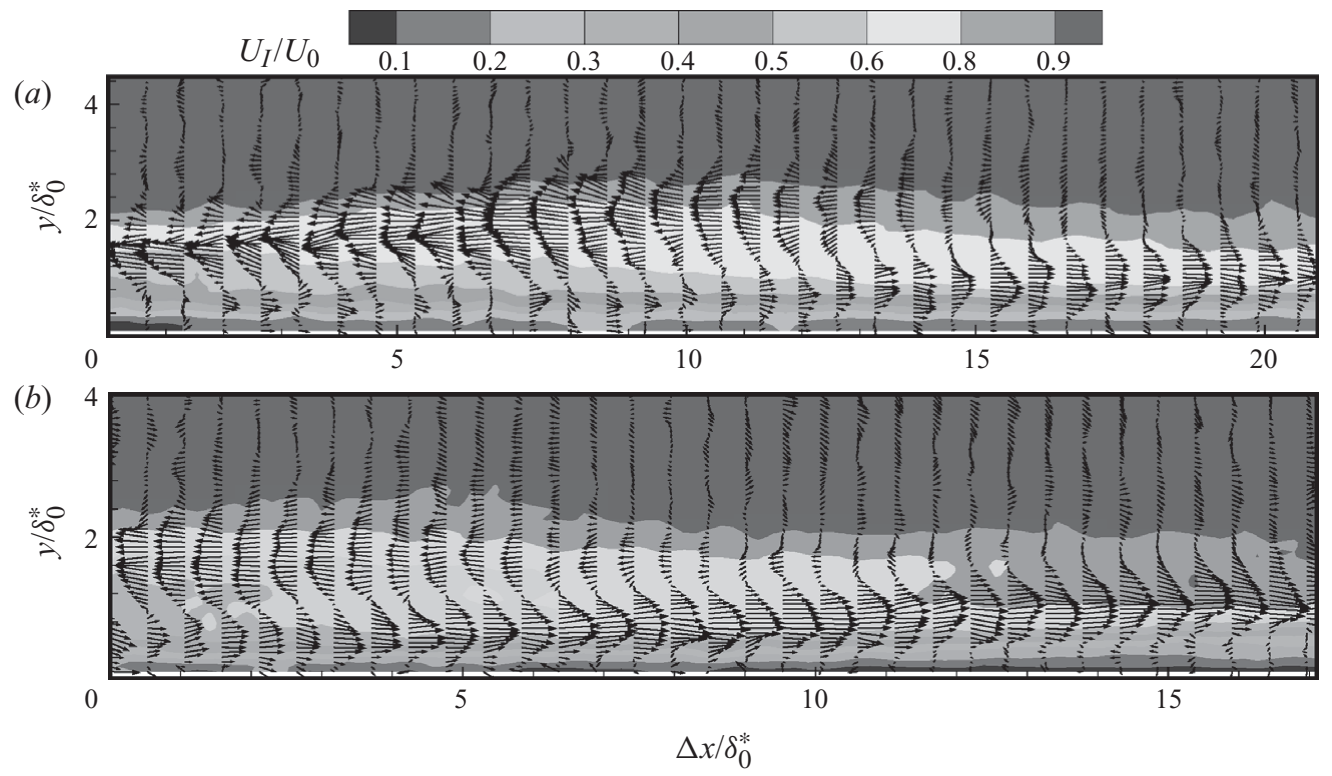

FIGURE 8. Simultaneous occurrence of both positive and negative $u$ fluctuations in a PIV realization. Fluctuating velocity vectors are plotted over the contours of the normalized instantaneous velocity, $U_{I} / U_{0}$. (a) Grid $1, \gamma \approx 1 \%$ flow and $(b)$ grid $3, \gamma \approx 1 \%$ flow.

Apart from the above observations, we also find that the positive and negative $u$ fluctuations can occur simultaneously in a single PIV realization. This is illustrated in figure 8 , for $\gamma \approx 1 \%$ flows. Simultaneous presence of these fluctuations may be attributed to the spanwise meandering motion of streaks. A low-speed and a highspeed streak, which are juxtaposed, may not be parallel to the streamwise direction due to the meandering motion, and the laser sheet in the wall-normal plane will cut both of these streaks simultaneously. Therefore, both these fluctuations will appear in a single PIV realization. Although the number of such frames in the entire PIV realizations was considerable, the values of $u / U_{0}$ for these fluctuations were found to be less than that for only positive $u$ fluctuations or only negative $u$ fluctuations.

Due to the prevalence of positive and negative $u$ fluctuations (figures 6 and 7) during transition, their individual contribution to the total root-mean-squared (r.m.s.) values of $u$ may be revealing. Therefore, the r.m.s. of the positive $u$ fluctuations, $u_{r m s, f}$, negative $u$ fluctuations, $u_{r m s, b}$, and that of the laminar-like PIV realizations, $u_{r m s, l}$, were obtained by separating them from the entire PIV realizations as follows. In each PIV realization, the number of data points showing positive and negative $u$ fluctuations and their magnitude at those data points were found first. We then used the magnitude and sign criteria to pick up a laminar-like frame or a particular $u$ fluctuation. The laminar-like frames were selected using the criterion, $-T u<u / U_{0}<+T u$, over $75 \%$ data points within a single frame. For the positive or negative $u$ fluctuations, if the magnitude of that $u$ fluctuation exceeded $5 \%$ of the free-stream velocity at large numbers of data points than the other $u$ fluctuations, it was considered for processing. For example, a positive $u$ fluctuation showing $u / U_{0} \geqslant 5 \%$ over $20-30 \%$ of the total data points and smaller fluctuations at other data points in the same PIV realization had been considered for the estimation of $u_{r m s, f}$. The wall-normal variations of $u_{r m s, f}$ and $u_{r m s, b}$ so obtained are shown in figures $9(a)$ and $9(b)$, along 

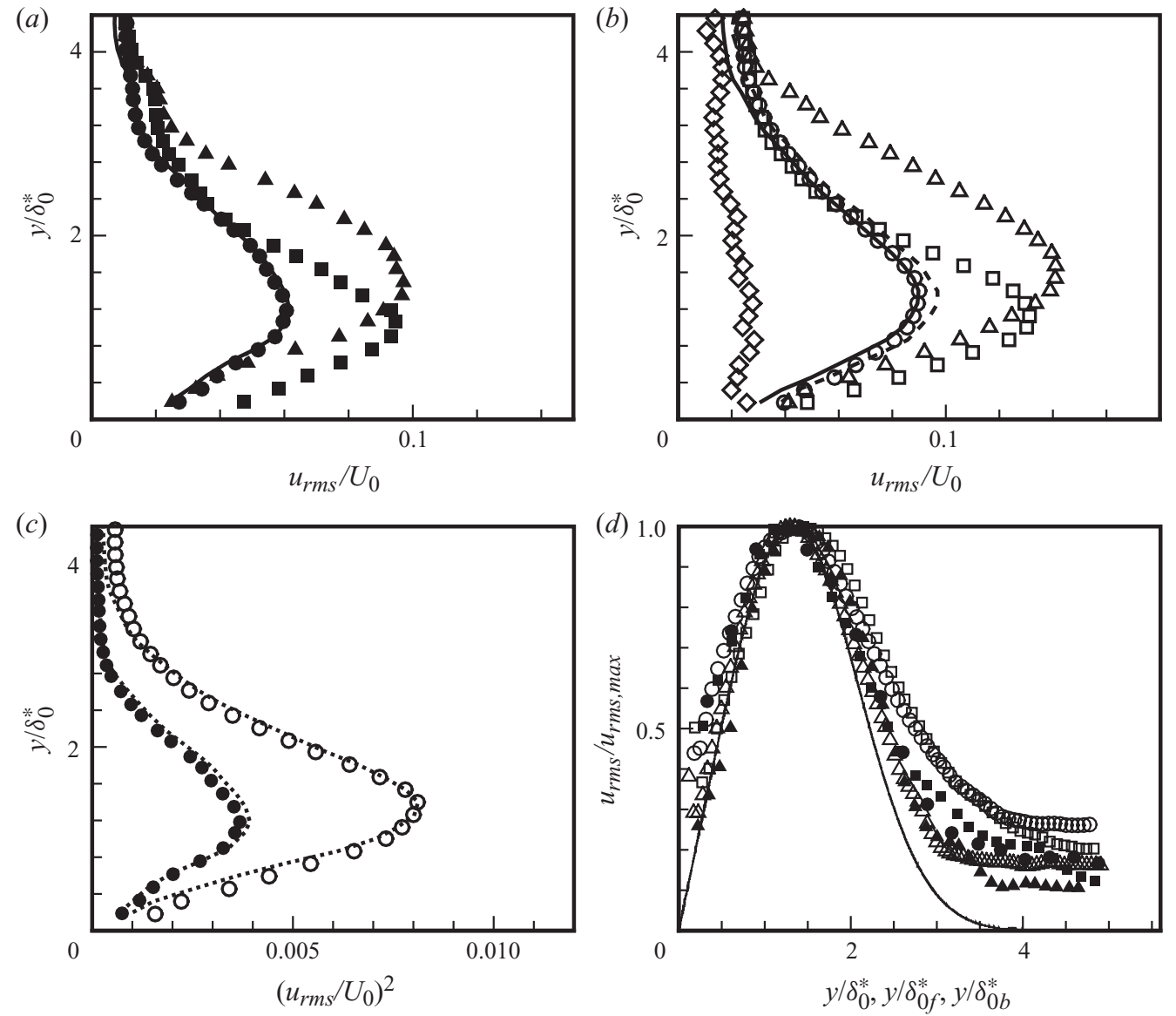

FIGURE 9. Distributions of r.m.s. of different $u$ fluctuations across the boundary layer. (a) Pre-transitional flows (filled symbols) for grid 1. (b) $\gamma \approx 1 \%$ flows (open symbols) for grid 3. Symbols: square, $u_{r m s, f}$; triangle, $u_{r m s, b}$; circle, $u_{r m s}$; diamond, $u_{r m s, l}$; solid line, $u_{r m s}=a\left(u_{r m s, b}+u_{r m s, f}\right), a \approx 0.34$; dashed line is the average of $u_{r m s, b}, u_{r m s, f}$ and $u_{r m s, l}$. (c) Comparison of measured $u_{r m s}^{2}$ (symbols) and linear combination (dotted line) $u_{r m s}^{2} \approx 2 a^{2}$ $\left(u_{r m s, f}^{2}+u_{r m s, b}^{2}\right)$ for the flows in $(a)$ and $(b) ; a \approx 0.34$. $(d)$ Comparison of normalized r.m.s. of various $u$ fluctuations in pre-transitional and transitional flows with non-modal theory (solid line) of Luchini (2000); symbols as in $(a)$ and $(b)$ except for laminar-like PIV realizations.

with the variation of $u_{r m s}$ in pre-transitional and $\gamma \approx 1 \%$ flows, for grids 1 and 3 , respectively. The distribution of $u_{r m s, l}$ for grid 3 is also displayed in figure $9(b)$. We find that the peak $u_{r m s, f}$ occurs in the inner half of the boundary layer, while the peak $u_{r m s, b}$ occurs in the outer half of the boundary layer. To be specific, the peak $u_{r m s, f}$ is found to be at $y / \delta_{0}^{*} \approx 1$ and the peak $u_{r m s, b}$ at $y / \delta_{0}^{*} \approx 1.65$. This is in agreement with the results of Hernon, Walsh \& Mceligot (2007), who found the peak of negative $u$ at $y / \delta \approx 0.6$ and the peak of positive $u$ at $y / \delta \approx 0.3$ from hot-wire measurements; here $\delta$ is their measured boundary-layer thickness. (We may mention that the first draft of this paper containing these results was submitted before Hernon et al. 2007.) However, Hernon et al. (2007) do not report such organized spatial structure of the fluctuating streamwise velocity component as presented here. The peak of negative $u$ seen in the outer half of the boundary layer is due to the negative $u$ fluctuations 


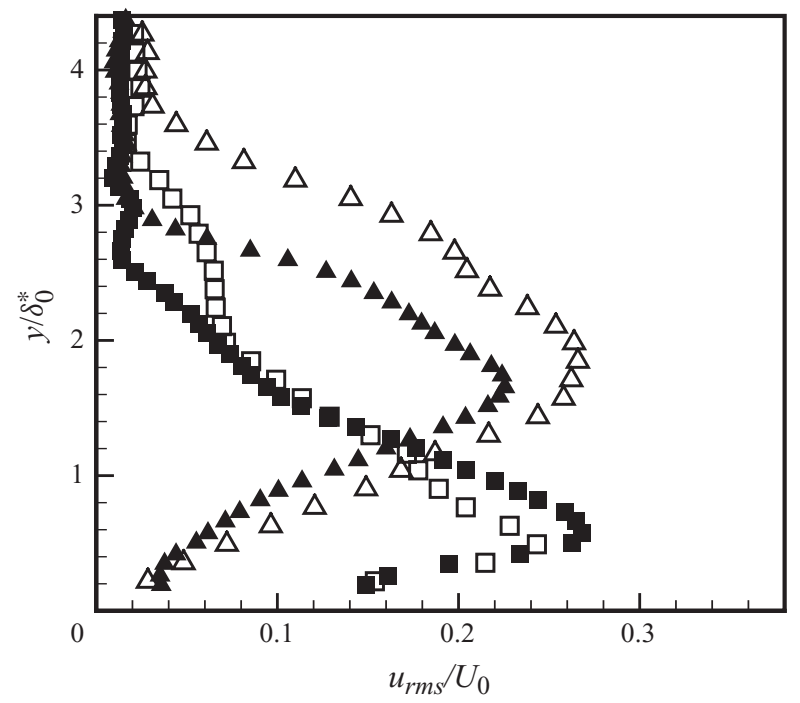

FIGURE 10. Distributions of r.m.s. of strong negative and positive $u$ fluctuations in $\gamma \approx 1 \%$ flows. Symbols: $\boldsymbol{\Lambda}, u_{r m s, b}$ and $\mathbf{\square}, u_{r m s, f}$ for grid $1 ; \Delta, u_{r m s, b}$ and $\square, u_{r m s, f}$ for grid 3 .

extending towards the boundary-layer edge. Similarly, the peak of positive $u$ is in the inner region due to the positive $u$ fluctuations dominating near the wall. However, it can be seen that the maximum values of $u_{r m s, f}$ and $u_{r m s, b}$ are nearly of the same order (about 1.35 times that of $u_{r m s}$ ), whereas $u_{r m s, l}$ is of the order of the free-stream turbulence intensity. The variations of $u_{r m s, l}, u_{r m s, f}$ and $u_{r m s, b}$ across the boundary layer suggest that the average of these quantities makes the peak of $u_{r m s}$ at nearly half the boundary-layer thickness, as shown in figure $9(b)$. Interestingly, we also find that $u_{r m s} \approx a\left(u_{r m s, f}+u_{r m s, b}\right)$, as shown by a solid line; here $a$ is a constant and its value is found to be approximately 0.34 , for both the grids. Since $u_{r m s, f}$ and $u_{r m s, b}$ are of the same order, it is possible that the energy, $u_{r m s}^{2}$, is also a linear combination of $u_{r m s, f}^{2}$ and $u_{r m s, b}^{2}$. We take, for example, $u_{r m s}^{2} \approx a^{2}\left(u_{r m s, f}+u_{r m s, b}\right)^{2}=2 a^{2}\left(u_{r m s, f}^{2}+\right.$ $\left.u_{r m s, b}^{2}\right)-a^{2}\left(u_{r m s, f}-u_{r m s, b}\right)^{2}$. Since the quantity $a^{2}\left(u_{r m s, f}-u_{r m s, b}\right)^{2}$ is very small, we have $u_{r m s}^{2} \approx 2 a^{2}\left(u_{r m s, f}^{2}+u_{r m s, b}^{2}\right)$, as shown in figure 9(c). Moreover, Matsubara \& Alfredsson (2001) have found that initially $\left(u_{r m s, \max } / U_{0}\right)^{2}$ grows linearly with $x$, for $\gamma \leqslant 20 \%$, which implies that the growth of $u$ is non-modal. Since $u_{r m s}$ is found to be a linear combination of $u_{r m s, f}$ and $u_{r m s, b}$, each one is expected to exhibit a nonmodal growth of Luchini (2000), as shown in figure $9(d)$. Note that the displacement thicknesses, $\delta_{0 f}^{*}$ and $\delta_{0 b}^{*}$, in the case of $u_{r m s, f}$ and $u_{r m s, b}$, respectively, were separately estimated from the respective mean velocities.

The maximum $u_{r m s}$ values can even be more than the values mentioned above. This information is lost when averaged over large samples. Andersson et al. (2001) have pointed out that the minimum amplitude of streak has to be, at least, $20 \%$ before breakdown. We separated those PIV realizations having $\left|u / U_{0}\right|>20 \%$ over several vectors of positive/negative $u$ fluctuations from the entire PIV realizations. These separated realizations were then averaged. The distributions of $u_{r m s, f}$ and $u_{r m s, b}$ across the boundary layer so obtained are shown in figure 10 , for $\gamma \approx 1 \%$ flows. It can be seen that the maximum $u_{r m s} / U_{0}$ can reach a value of about 0.25 in the $\gamma \approx 1 \%$ flow, which is in the early state of breakdown. This figure also shows that the peak $u_{r m s, f}$ occurs at around $y / \delta_{0}^{*} \approx 0.53$ and the peak $u_{r m s, b}$ is at around $y / \delta_{0}^{*} \approx 1.75$. 

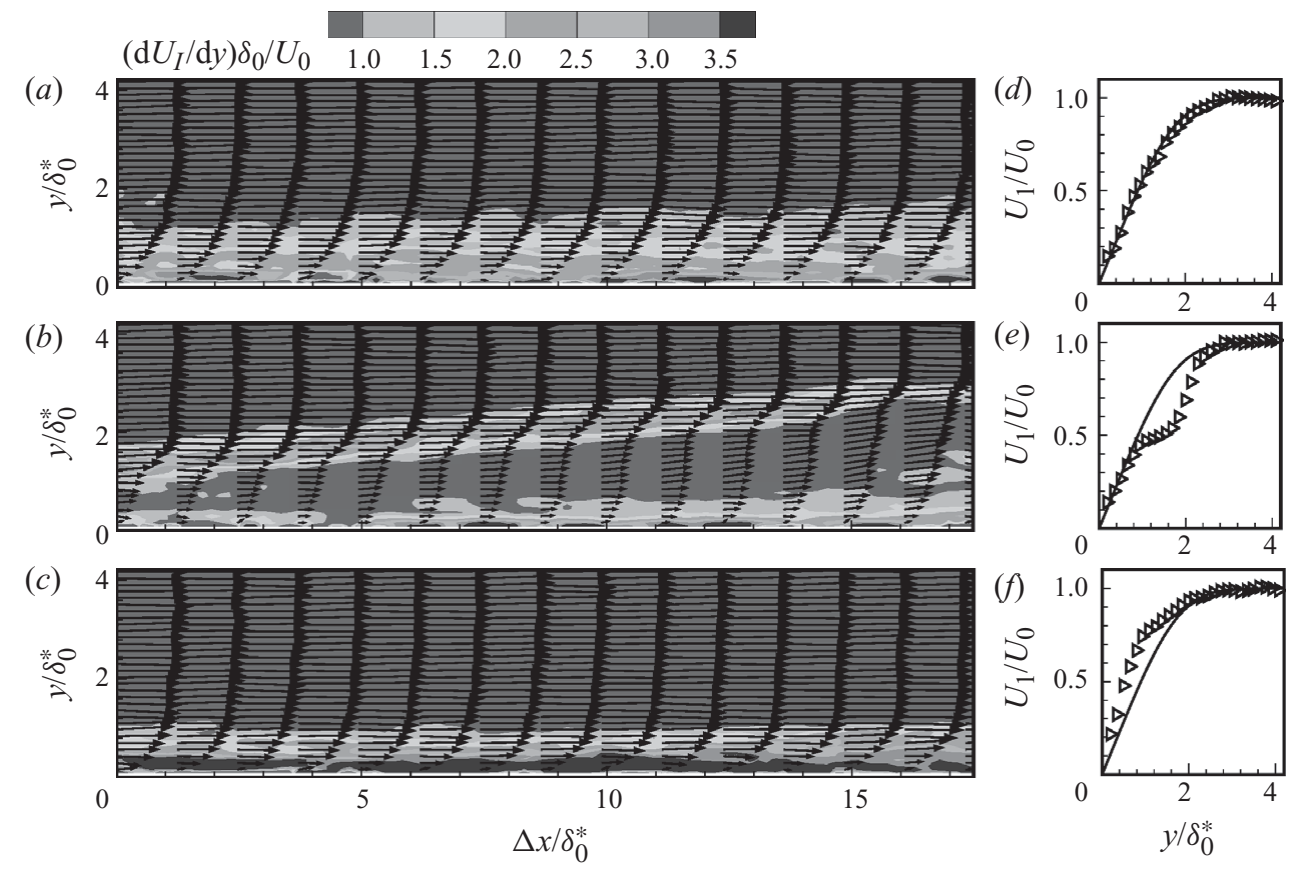

FIGURE 11. Instantaneous velocity distribution across the boundary layer for the frames in figure 7 and comparison with the Blasius profile. $(a-c)$ Instantaneous velocity vectors over the contours of normalized instantaneous shear, $\left(\mathrm{d} U_{I} / \mathrm{d} y\right) \delta_{0} / U_{0} .(d-f)$ Comparison of measured velocity profiles $(\triangleright)$ at $\Delta x / \delta_{0}^{*} \approx 10$ (in $a-c$ ) with the Blasius profile, $-\gamma \approx 1 \%$ flow, grid 3 .

Thus far, we have mostly presented various organized $u$ fluctuations in freestream turbulence-induced transitional flows. However, it may be of interest to study the instantaneous velocity profiles corresponding to these fluctuations. For the PIV realizations in figure 7 , the corresponding instantaneous velocity vectors are displayed in figure $11(a-c)$, respectively. A comparison of the velocity profile at $\Delta x / \delta_{0}^{*} \approx 10$ with the Blasius profile is also shown in figure $11(d-f)$. The instantaneous velocity vectors in figure 11(a) show a laminar-like velocity field, which is clearly seen in figure $11(d)$. This may be expected as the velocity fluctuations inside the boundary layer are almost of the same order of the free-stream fluctuations, as mentioned earlier. For the negative $u$ fluctuations, figures $11(b)$ and $11(e)$ show that the corresponding instantaneous velocity field is highly inflectional in the inclined shear-layer region. Therefore, the lifted-up negative $u$ fluctuations are also associated with the instantaneous inflectional velocity profile. Figures $11(c)$ and $11(f)$ show fuller instantaneous velocity profiles due to the associated positive $u$ fluctuations. Similar instantaneous inflectional velocity profiles, along with inclined shear layers, were also observed for other grids considered in this study; figure 12 illustrates this for grids 1 and 2 in $\gamma \approx 1 \%$ flows. The present experimental results showing the presence of the inflectional velocity profile and inclined shear layer in an actual bypass transition are interesting and new.

Since our measurements are not time resolved, an exact breakdown scenario remains elusive. However, it appears that the lifted-up high shear layer undergoes instability and starts shedding vortices at the outer edge of the boundary layer, as can be deduced from figures 12 and 13. Towards the end of the frame in figure 13(a), we also observe a kink-like pattern in the lifted-up oscillating shear layer at the 

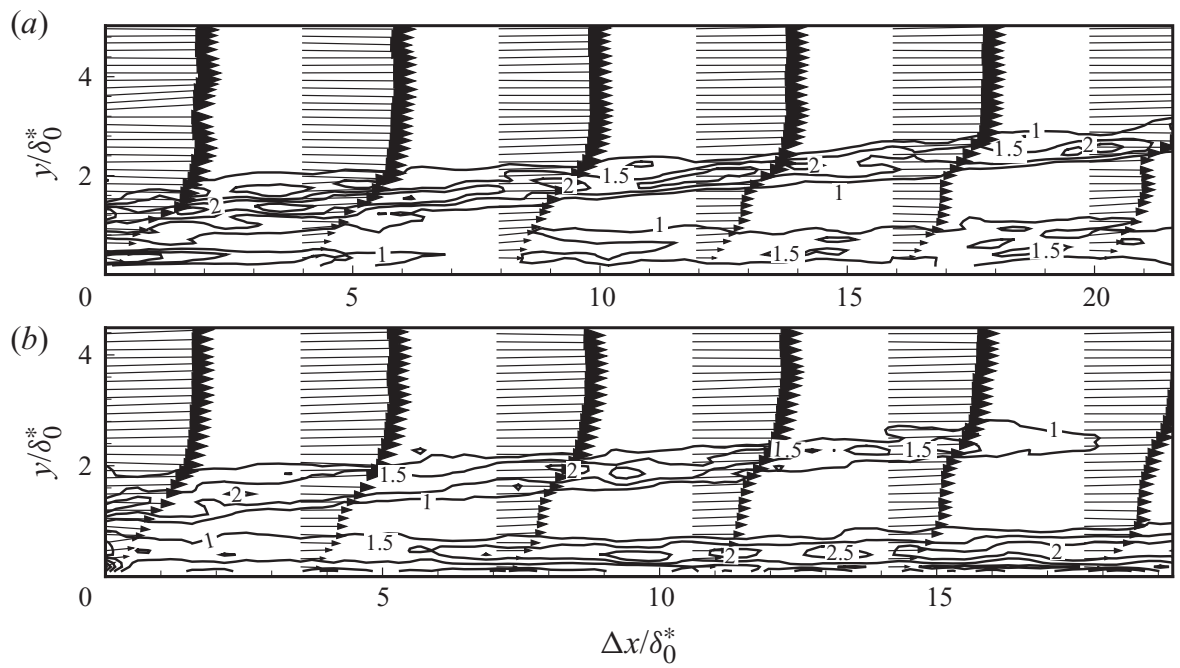

FigURE 12. Non-dimensional instantaneous iso-shear lines of $\left(\mathrm{d} U_{I} / \mathrm{d} y\right) \delta_{0} / U_{0}$. Arrows represent the instantaneous velocity vectors. (a) Grid $1, \gamma \approx 1 \%$ flow and $(b)$ grid $2, \gamma \approx 1 \%$ flow.
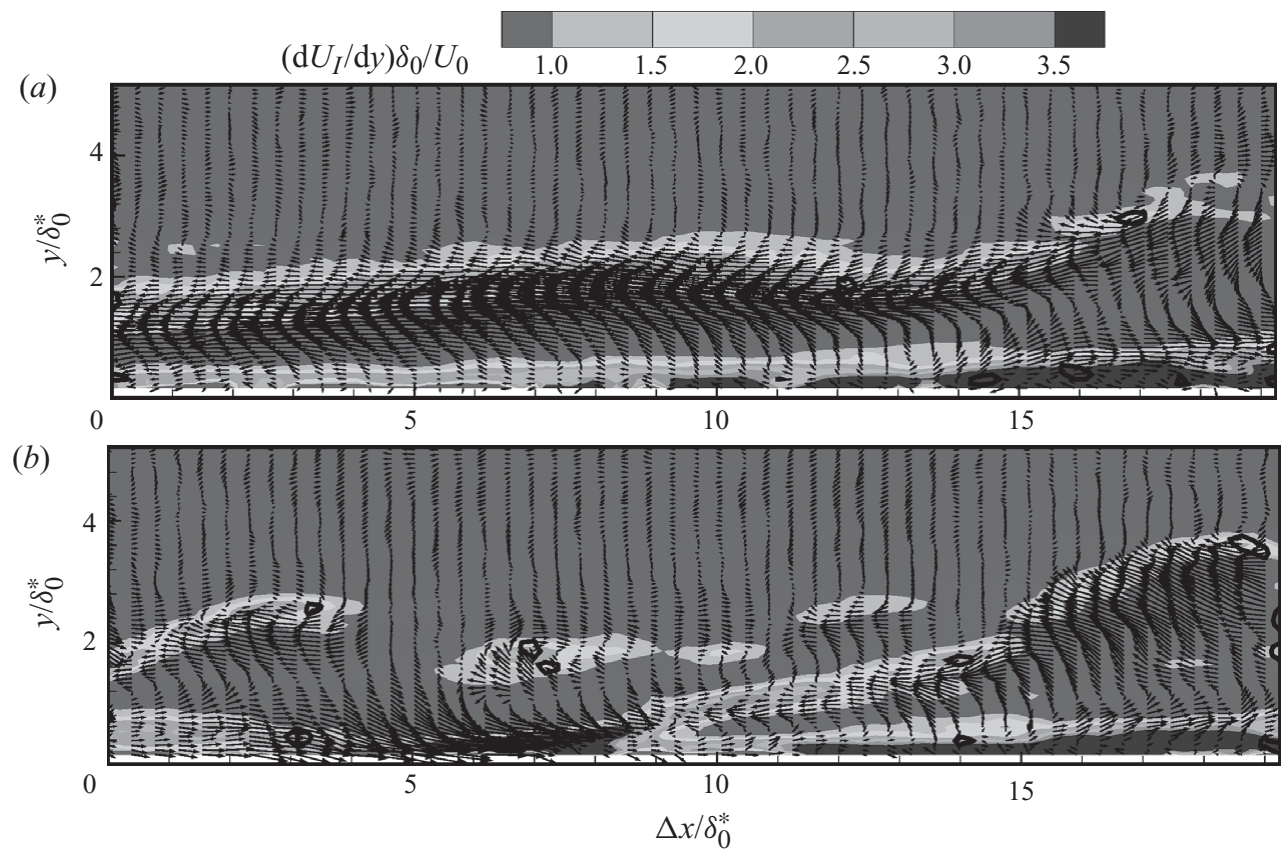

FiguRE 13. Lift-up of shear layer and its oscillation. Grey levels indicate normalized instantaneous shear, $\left(\mathrm{d} U_{I} / \mathrm{d} y\right) \delta_{0} / U_{0}$; line contours represent swirl strength and vectors are fluctuating velocity vectors. $\gamma \approx 20 \%$ flow, grid 3 .

boundary-layer edge. Lifted-up high-shear layers with kinks are the characteristics of the secondary instability (e.g. Nishioka et al. 1981; Rist \& Fasel 1995). In their controlled study of late stages of transition in Poiseuille flow, Nishioka et al. (1981) found that the kink in the high-shear layer corresponds to the beginning of the 
vortex roll-up process and a hot-wire senses a large low velocity pulses as the kink passes it. Similar vortex roll-up process with negative $u$ can be seen in the upper right side of the inclined shear layer in figure 13(a). In order to identify the vortices in this figure, we applied the criteria of Chong, Perry \& Cantwell (1990). For a three-dimensional flow field, Chong et al. (1990) found that if the discriminant of the characteristic equation of a local velocity gradient tensor is positive, its one eigenvalue is positive and other two are complex conjugate. In that case, the particle trajectories around the eigenvector corresponding to the real eigenvalue will show swirling, spiral motion. In the absence of three-dimensional data, an equivalent two-dimensional velocity gradient tensor from two-dimensional PIV data may also be used to identify vortices (Adrian, Christensen \& Liu 2000a). Imaginary part of the complex eigenvalue of the local velocity gradient tensor is the swirl strength which shows the location of vortices. Moreover, this criterion is independent of the convection velocity of the vortex (Adrian et al. 2000a). The line contours in figure 13 represent the swirl strength and show the location of the vortices. The shear layers in figures 13(a) and 13(b) resemble somewhat those of Nishioka et al. (1981) at one-spike and three-spike stages (see their figures 4 and 5), respectively. The near-wall thin shear layer in the region $5 \leqslant \Delta x / \delta_{0}^{*} \leqslant 8$ in figure $13(b)$ also resembles that of Nishioka et al. (1981), which they speculate to be turbulent wall phenomena.

While it remains to confirm whether a spot appears near the boundary-layer edge or near the wall, spot-like features were seen in several PIV realizations. A flow breakdown scenario at $\gamma \approx 20 \%$ flow (for grid 3 ) is shown in figure 14 . The vectors in figure 14(a) represent the fluctuating velocity field in the foreground of $v$ velocity: darker region, $v<0$; light white, $v>0$. Corresponding to this frame, the nondimensional instantaneous vorticity $\left(\Omega_{z} \delta_{0} / U_{0}\right)$ contours are shown in figure $14(b)$, and $u / U_{0}$ contours in figure 14(c). Figure 14(a) shows that a strong positive $u$ near the wall seems to force the relatively low speed fluid out from the plate. A strong negative $v$ may also be seen in the region $5 \leqslant \Delta x / \delta_{0}^{*} \leqslant 10$. The fluctuating velocity vectors also show two vortices of opposite sign in the region $5 \leqslant \Delta x / \delta_{0}^{*} \leqslant 8$. This vortical feature was found to appear consistently with this kind of PIV realization. The line contours in figure 14(a) represent the swirl strength. It can be seen that their locations also match with the vorticity locations in figure $14(b)$. As the vortices are moving at different convection velocities, they are not perfectly captured by the fluctuating velocity vectors in the present Reynolds decomposition. If the lifted-up high shear layer is the spot precursor, then this vortical feature may be considered as the shed vortices from this unstable shear layer (see figure 13) leading to a turbulent spot. We also note that the maximum value of $u / U_{0}$ was found to be higher than $30 \%$, whenever we encountered this type of breakdown-like frames (figure $14 c$ ). The two important flow features worth noting here are the following. In the region $4 \leqslant \Delta x / \delta_{0}^{*} \leqslant 10$, one finds a layer of negative $u$ sitting on a layer of positive $u$ that continues upstream (figure 14c). The second one is the detached vortices of significant strength in the outer part of the boundary layer (see figure 14b). These two features have been seen in artificial turbulent spots studied by others (e.g. Wygnanski, Zilberman \& Haritonidis 1982; Singer 1996). For instance, Singer (1996) reported these features in his direct numerical simulation of turbulent spot (see his figures $2 a$ and 4). The observed near-wall vorticity layers and the calm region of positive $u$ up to $\Delta x / \delta_{0}^{*} \approx 8$ are also seen in the simulated turbulent spot (Singer 1996). Therefore, the present breakdown scenario represents a turbulent spot. In $\gamma \approx 1 \%$ flows, the breakdown picture was found to be similar (Mandal et al. 2006). The number of such frames was found to increase with increasing flow intermittency. Such a similarity 

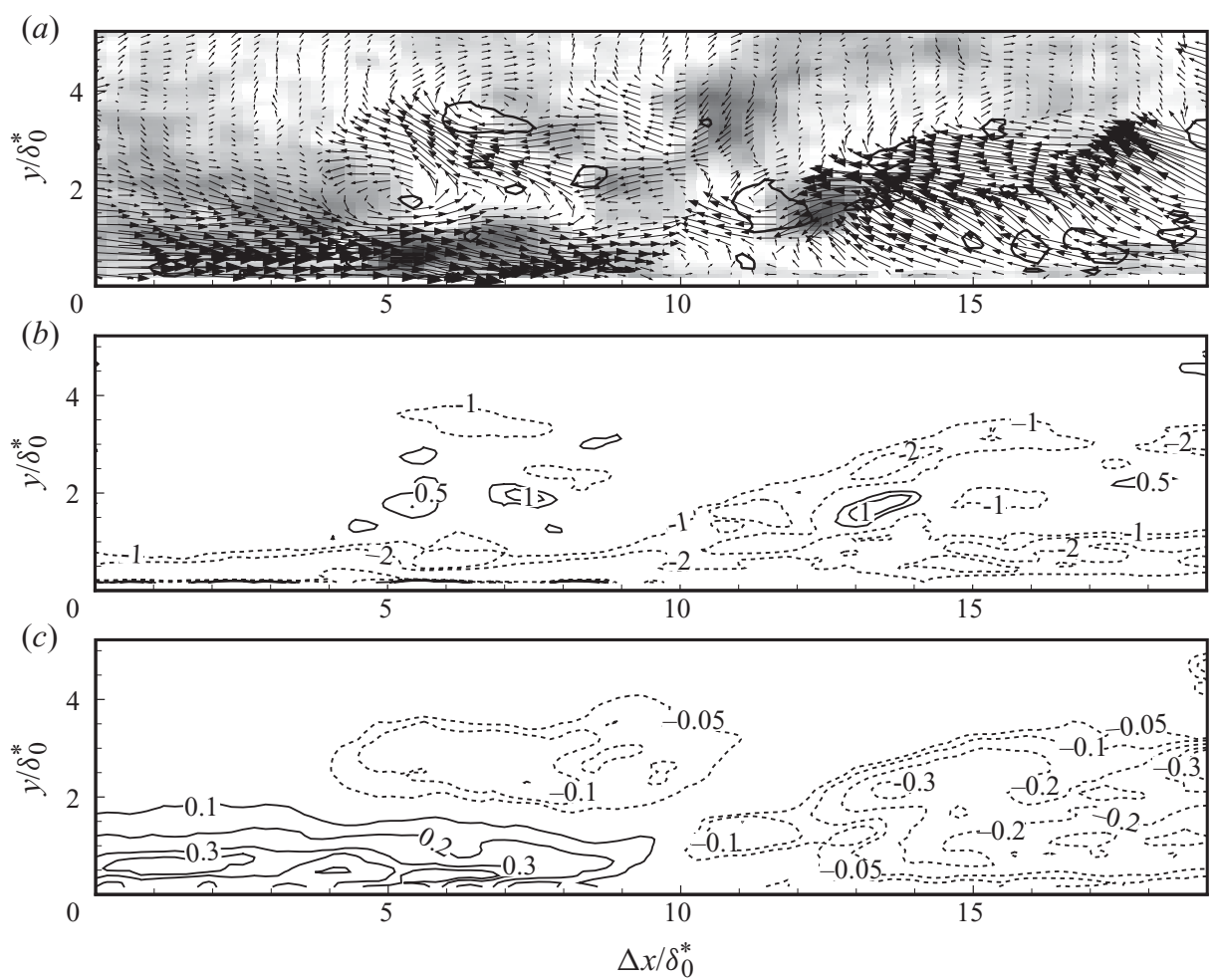

FIGURE 14. Flow breakdown scenario in the $\gamma \approx 20 \%$ flow (grid 3). (a) Fluctuating velocity vectors in the foreground of instantaneous $v$ : dark, $v<0$; light white, $v>0$; line contour represents swirling strength. $(b)$ Non-dimensional instantaneous vorticity $\left(\Omega_{z} \delta_{0} / U_{0}\right)$ contours: full line, positive value; dashed line, negative value. (c) Contour values are $u / U_{0}$; full line, positive value; dashed line, negative value.

with an artificial turbulent spot in an actual transition is not yet reported in the available literature.

\subsection{Spanwise plane}

The spanwise PIV measurements were carried out in the wall-parallel plane at $y / \delta_{0} \approx 0.5$ and at the streamwise locations mentioned earlier. In order to confirm that the PIV results are comparable with other results, the average streak spacing was estimated from the spatial correlation function, $R_{u u}$, as shown in figure 15 , for pretransitional and $\gamma \approx 1 \%$ flows. The streak spacing was taken as twice of the distance at which the first minimum of the correlation function occurs (Jacobs \& Durbin 2001; Matsubara \& Alfredsson 2001). The present streak spacing of approximately $1.9 \delta_{0}$ and $1.4 \delta_{0}$ in pre-transitional and $\gamma \approx 1 \%$ flows, respectively, compare well with the other results (e.g. Westin et al. 1994; Jacobs \& Durbin 2001; Matsubara \& Alfredsson 2001).

Figure $16(a-d)$ shows the representative high-speed and low-speed streaks in $\gamma \approx 1 \%$ flows, for grids 1 and 3 . In addition to almost straight streaks in the streamwise direction (see figures $16 a$ and $16 c$ ), some streamwise oscillating streaks are also seen in figures $16(b)$ and $16(d)$. The meandering motion of streaks may be inferred from these figures, as a particular streak does not stay at the same position in the subsequent PIV realizations and sways in the spanwise plane (see figure $16 d$ ). The 


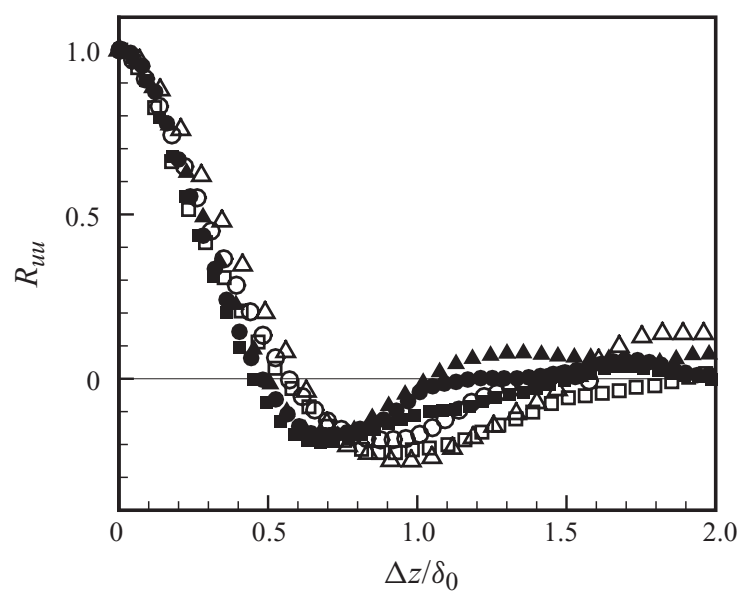

FIGURE 15. Spanwise correlation function for pre-transitional and $\gamma \approx 1 \%$ flows, for all three grids. These results are based on an average of around 500 PIV realizations in the spanwise plane. Pre-transitional flows at $x_{1}=310 \mathrm{~mm}: \triangle$, grid $1 ; \square$, grid $2 ; \bigcirc$, grid 3 . The $\gamma \approx 1 \%$ flows at $x_{1}=460 \mathrm{~mm}: \mathbf{\Delta}$, grid $1 ; \mathbf{\square}$, grid $2 ; \boldsymbol{\bullet}$, grid 3 .
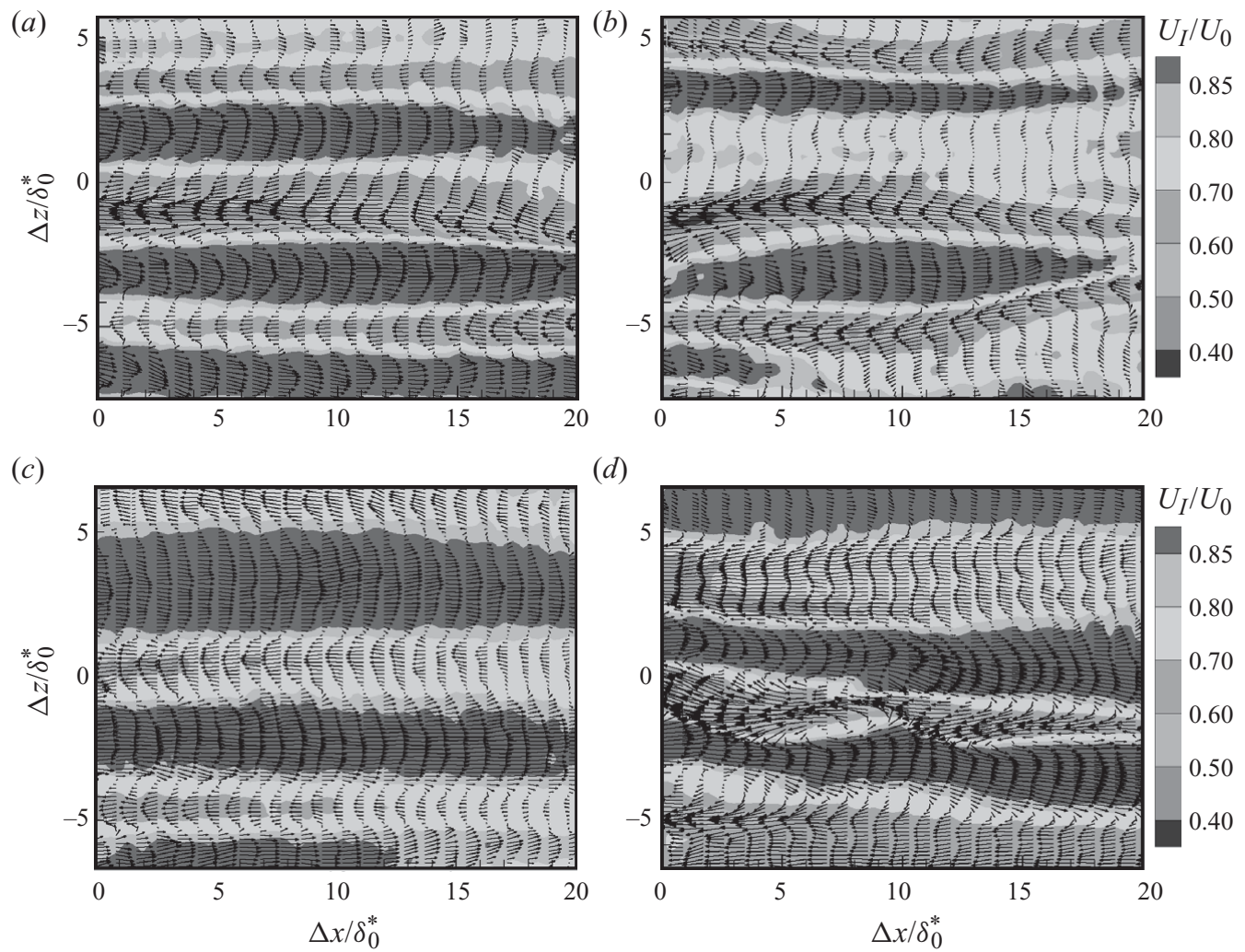

FIGURE 16. Four instantaneous PIV realizations depicting low- and high-speed streaks in the spanwise plane. The fluctuating velocity vectors of $u$ and $w$ are plotted over the contours of normalized instantaneous streamwise velocity. $(a-b) \gamma \approx 1 \%$, grid $1 ;(c-d) \gamma \approx 1 \%$, grid 3 .

fluctuating velocity vectors of $u$ and $w$ in these figures exhibit that the positive and negative $u$ fluctuations appear in a comparatively high and low instantaneous velocity regions, respectively, and therefore the corresponding fluctuations can clearly represent 

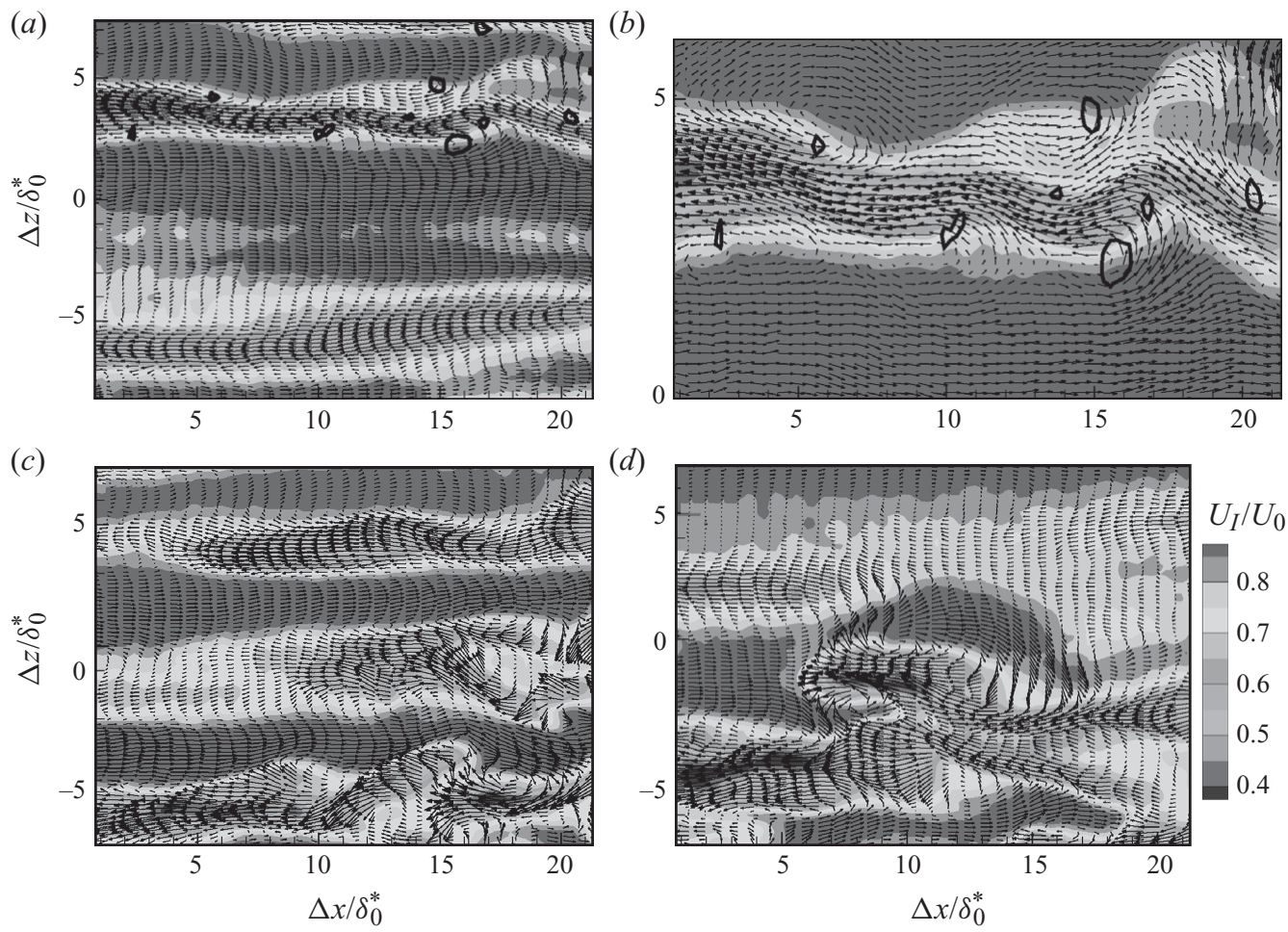

FIGURE 17. Instantaneous PIV realizations illustrating possible streak breakdown process in the spanwise plane. The fluctuating velocity vectors of $u$ and $w$ are plotted over the contours of the normalized instantaneous streamwise velocity. (a) Sinuous oscillation of a low-speed streak. Line contours: swirl strength. (b) Zoomed view of $(a)$. (c) Varicose-type oscillation of a low-speed streak. $(d)$ Interaction of streaks. $\gamma \approx 1 \%$ flow, grid 3 .

high- and low-speed streaks in bypass transition, as mentioned earlier. Moreover, these fluctuating velocity vectors appear similar to the simulated results of Jacobs \& Durbin (2001). The appearance of these jet-like structures of the fluctuating velocity components seems to be associated with streak unsteadiness. Since streaky structures meander in the spanwise plane, the local mean velocity becomes independent of the spanwise distance and therefore $u(x, y, z, t)=U_{I}(x, y, z, t)-\bar{U}(x, y) \neq 0$. If streaks are steady, they will remain at the same location and the local mean velocity becomes a function of all the three space coordinates. In this case, $U_{I}(x, y, z, t) \approx \bar{U}(x, y, z)$, for instance, and the fluctuating velocity, $u(x, y, z, t)$, will be approximately zero. That is, the presence of streaks may not necessarily guarantee the presence of the jet-like structure of the fluctuating velocities. In a recent study by Banerjee et al. (2006), where streaks were triggered by a small amplitude disturbance in a flat-plate boundary layer, no jet-like structure of the fluctuating velocity components was found at their first measurement location, as the streak was almost steady there. Further downstream, where streaks began to be unsteady, a jet-like structure of the fluctuations was observed.

Several PIV realizations in $\gamma \approx 1 \%$ flows also revealed relatively high frequency oscillations of some streaks compared to those in figures $16(b)$ and $16(d)$. Figure $17(a)$ shows sinuous oscillation of a low-speed streak with increasing amplitude in the downstream direction. This is better seen in the zoomed view in figure 17(b). Black thick lines in this figure represent the contour of the swirl strength, which identifies 

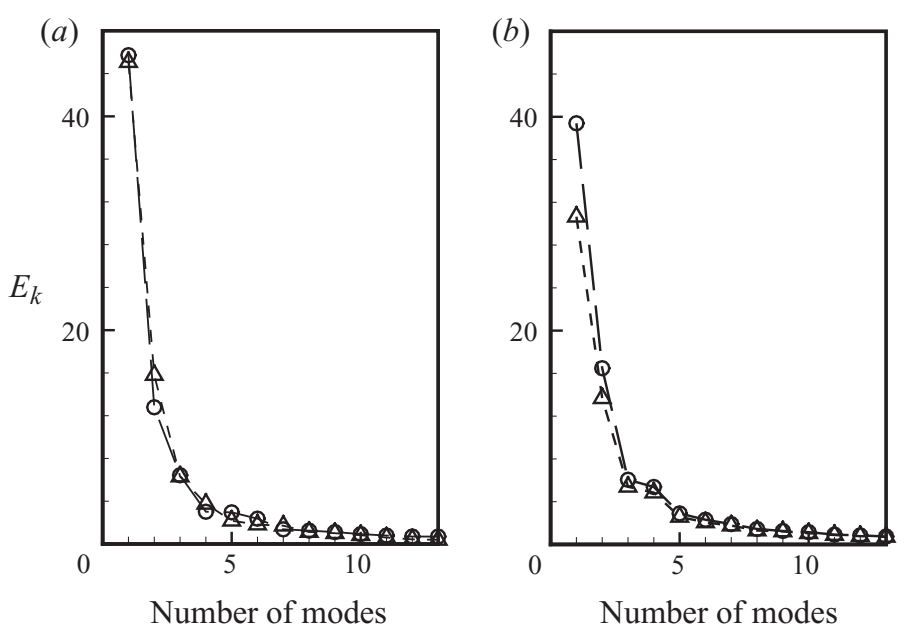

FIGURE 18. Relative energy associated with various POD modes. (a) Pre-transitional flows;

(b) $\gamma \approx 25 \%$ (grid 1) and $20 \%$ (grid 3) flows. Symbols: - - $\triangle--$, grid $1 ;-\bigcirc-$, grid 3.

the vortex locations along the oscillating low-speed streak. A staggered pattern of these contours along the low-speed streak is clearly seen. The simulation by Brandt \& Henningson (2002) shows a staggered pattern of quasi-streamwise vortices on the flanks of sinuous low-speed streaks, both inclined away from the wall and tilted in the downstream direction. Therefore, the present vortices in figure 17(b) may be the cross-section of these inclined and tilted quasi-streamwise vortices. Two additional contours of relatively bigger diameter at around $\Delta x / \delta_{0}^{*}=15$ may be due to the roll-up structures (e.g. Mans et al. 2005) seen towards the end of the oscillating low-speed streak. Somewhat varicose-type modes are also seen in figures $17(c)$ and $17(d)$ (see the low-speed streak in the region $-3 \leqslant \Delta z / \delta_{0}^{*} \leqslant 0$ in figure $17(c)$ and the low-speed streak below the centreline in figure $17 d$ ). The engulfing of a portion of a low-speed streak by a high-speed streak at around $\Delta z / \delta_{0}^{*}=-2$ is also visible in figure $17(d)$. Brandt et al. (2004) suggest that the interaction between a low- and high-speed streak plays an important role in the formation of an incipient turbulent spot. The importance of such an interaction in sinuous breakdown was also noted by Mans et al. (2007), who consider this as the discontinuity in the streak pattern. Since the wall-normal shear and inflectional velocity profiles are closely related to the varicose type of oscillation (e.g. Brandt et al. 2004), such an interaction (figure 17d) may also contribute to the breakdown process mentioned in $\S 4.1$.

\section{POD results}

The results in this section are based on sufficient number of snapshots providing nearly converged eigenvalues (e.g. Cazemier, Verstappen \& Veldman 1998). Figure 18 shows the percentage of the relative energy, $E_{k}$, associated with each POD mode in pre-transitional, $\gamma \approx 20 \%$ (grid 3) and $\gamma \approx 25 \%$ (grid 1) flows. The peak energy is seen to reduce in $\gamma \approx 20 \%$ and $25 \%$ flows and the energy of some lower modes is enhanced, compared to the pre-transitional flows. Figure $18(b)$ also shows that the third and fourth modes have almost the same relative energy. That is, the eigenvalues corresponding to these modes are near degenerate and we have two eigenfunctions for nearly the same eigenvalue. Degenerate eigenspectrum is also reported in other 


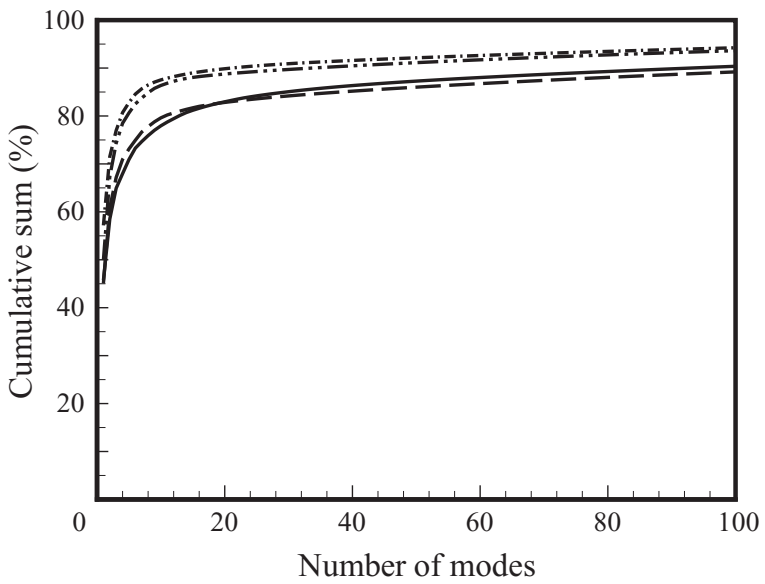

FIGURE 19. Cumulative energy distribution in pre-transitional flows for grids 1 and 3 . Lines:

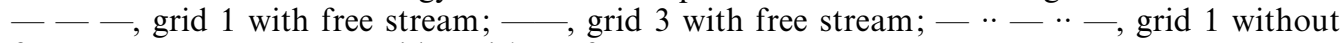
free stream; - - - - _ grid 3 without free stream.

flows (e.g. Deane et al. 1991; Gunes, Liakopoulos \& Sahan 1997) including transitional boundary-layer flows (e.g. Rempfer \& Fasel 1994; Gunes \& Rist 2004), and are attributed to the travelling waves, as discussed in detail later in this section. We may note that these results are based on the data set extending up to $7-8 \delta_{0}^{*}$ in the wall-normal direction, and thus a portion of the free-stream flow is included in the present analysis (e.g. Rempfer \& Fasel 1994). However, when this was excluded by considering the data set up to $y / \delta_{0}^{*} \approx 3.5$, the energy convergence improved, as shown for pre-transitional flows in figure 19. This figure also shows that most of the energy is captured by the first few modes; with and without free-stream fluctuations, the first 10 modes capture $78 \%$ and $88 \%$ of the total energy, respectively (figure 19). Therefore, the dynamics of bypass transition appear to be a low-dimensional one.

A POD eigenvalue can be considered as a measure of the mean value of the energy that the corresponding mode contributes to the flow (Rempfer \& Fasel 1994). Figure 20 shows the first four modes corresponding to the first four eigenvalues contributing $68 \%$ and $67 \%$ of the total energy in the pre-transitional and $\gamma \approx 20 \%$ flows (grid 3), respectively. Since eddies are often composed of many modes, an individual mode itself cannot describe the structure of an eddy. Despite the fact that several POD modes may be needed to describe the structure of an eddy, the structure of each mode may also be of interest as a component of the complete structure (Liu et al. 2001). Although the first mode in figures 20(a) and 20(b) shows positive $u$ fluctuations, it may be a representative of either positive or negative $u$ fluctuations depending on the sign of the coefficient, $a^{1}\left(t_{n}\right)$, for this mode. However, defining a structure as a combination of the first two POD modes, i.e. $\boldsymbol{\Psi}\left(\boldsymbol{x}, t_{n}\right)=\sum_{k=1}^{2} a^{k}\left(t_{n}\right) \boldsymbol{\Phi}^{k}(\boldsymbol{x})$, a positive or negative $u$ fluctuation may be represented even better and closer to those seen in the instantaneous PIV realizations. For example, if the first and second modes in figure 20(b), with properly weighted positive coefficients, are added, a near-wall positive $u$ fluctuation can be obtained satisfactorily than the first mode alone. Similarly, the negative $u$ fluctuations away from the wall is better represented with properly weighted negative and positive coefficients. The combination of the first and second modes can thus represent $u$ fluctuations similar to those seen in the instantaneous realizations. The third and fourth modes contain 

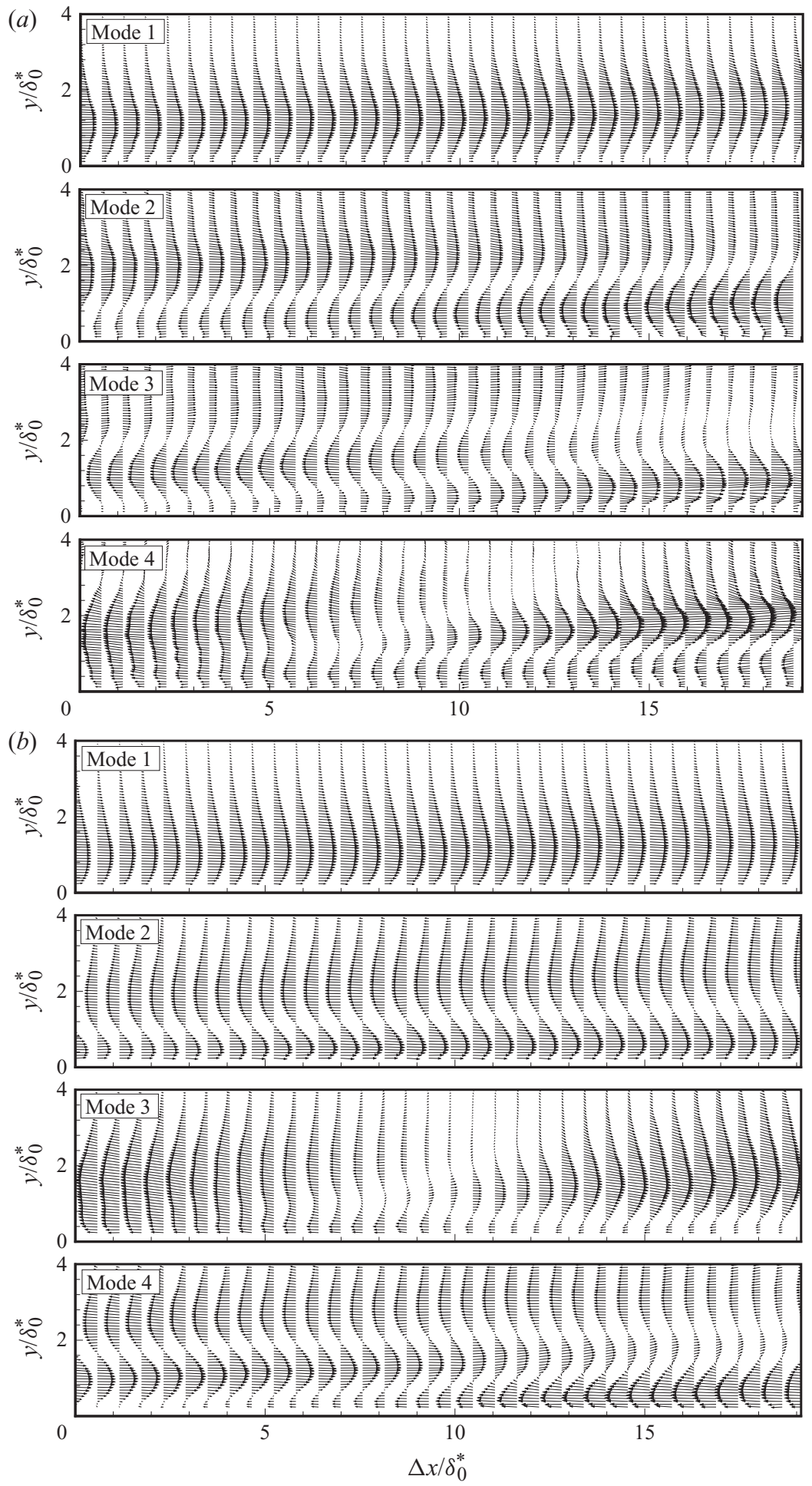

FIGURE 20. First four energetic POD modes for grid 3. (a) Pre-transitional flow; (b) $\gamma \approx 20 \%$ flow. 

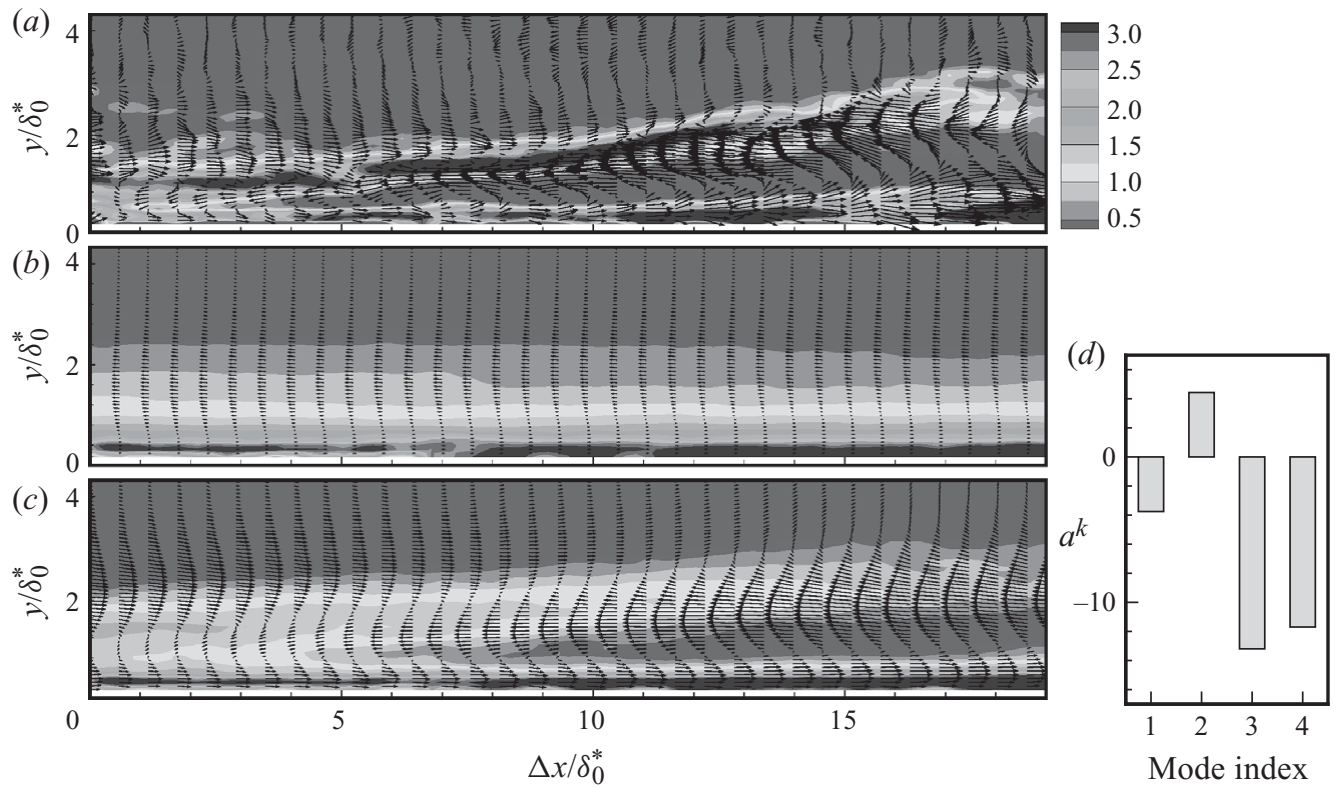

FigURE 21. Reconstruction of an instantaneous PIV realization at $x_{1}=460 \mathrm{~mm}$ in the $\gamma \approx 20 \%$ flow, for grid 3; contour shows normalized instantaneous shear, $\left(\mathrm{d} U_{I} / \mathrm{d} y\right) \delta_{0} / U_{0}$. (a) An actual fluctuating velocity field with an inclined shear layer. (b) Two-mode reconstruction. (c) Four-mode reconstruction. $(d)$ Bar plot of the coefficient, $a^{k}$, of each mode for this reconstruction.

mainly two opposite $u$ fluctuations and each occupies nearly a half of the streamwise extent considered here. These modes may arise due to the streamwise waviness of streaks or due to their swaying in the spanwise plane. For example, consider the oscillating low-speed streak around the centre of the frame in figure 16(d). A laser sheet in the wall-normal plane measurement may cut both low-speed and highspeed streaks simultaneously, leading to two opposite $u$ fluctuations in the same PIV realization. However, for a better understanding of the manifestation of these modes, a flow reconstruction was carried out in the $\gamma \approx 20 \%$ flow (grid 3), using (3.3) with the coefficients, $a^{k}\left(t_{n}\right)$, obtained from (3.4). Figure 21( $\left.a\right)$ shows an instantaneous snapshot containing an inclined shear layer; vectors are fluctuating velocity vectors. The two-mode reconstruction in figure $21(b)$ recovers small negative $u$ fluctuations only, although both negative and positive $u$ fluctuations are present in figure $21(a)$. However, the four-mode reconstruction in figure 21(c) shows a better approximation of the instantaneous picture, including the inclined shear layer; the instantaneous shear was estimated using the mean velocity and the reconstructed streamwise fluctuating velocity, i.e. $\partial(\bar{U}+u) / \partial y$. The contribution of a particular mode in the reconstruction can be obtained from the magnitude of the coefficient of that mode, as shown by the bar plot of $a^{k}$ in figure $21(d)$. The major contributions are seen to come from the third and fourth modes in this four-mode reconstruction. In general, the contribution of these two modes were found to be significant for those PIV realizations containing inclined shear layers (e.g. figures 13, 14 and 21). Higher modes, although contain a high level of details, are not shown here, as there may be an upper limit for flow details (Pedersen \& Meyer 2002). On the whole, only a few energetic POD modes can depict the main bypass transition events. 

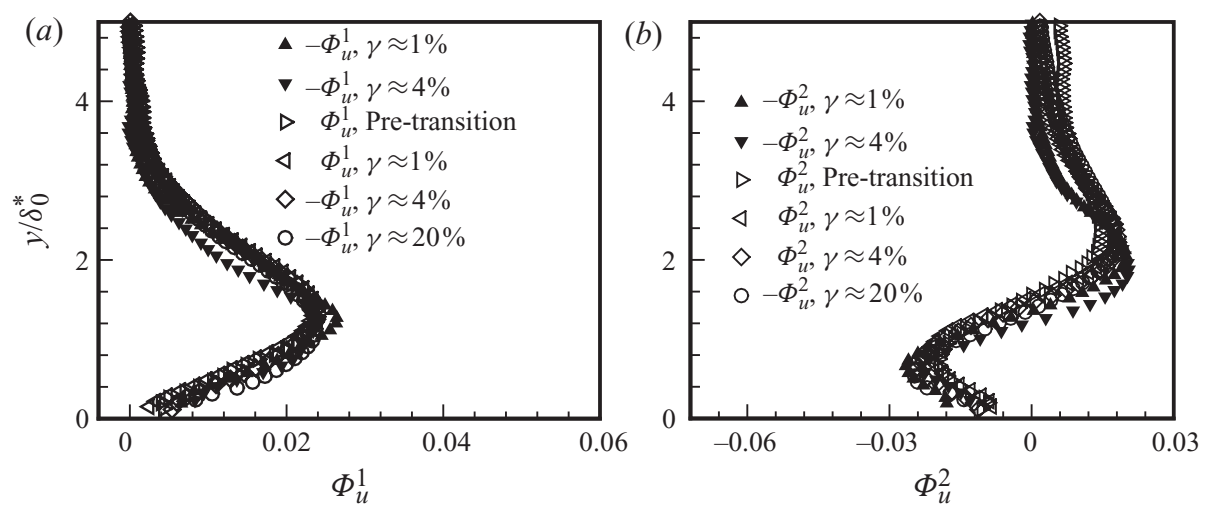

FIGURE 22. Wall-normal variation of the $u$ component corresponding to the first two POD modes, $\Phi_{u}^{1}$ and $\Phi_{u}^{2}$, for pre-transitional, $\gamma \approx 1 \%, \gamma \approx 4 \%$ and $\gamma \approx 20 \%$ flows. Filled symbols, grid 1 ; open symbols, grid 3. (a) $-\Phi_{u}^{1}$, plots on $+\Phi_{u}^{1}$ for mode $1 ;(b)-\Phi_{u}^{2}$ plots on $+\Phi_{u}^{2}$ for mode 2 .

The first POD mode in figure 20(a) is similar to the corresponding POD mode in figure 20(b). Similarity is also noticed for the second POD mode in the corresponding figures, except for the sign of $u$ fluctuations. Figure 22 shows the wall-normal variation of $u$ component corresponding to these two POD modes. One may notice that, except for the sign, the corresponding modes are almost similar in pre-transitional, $\gamma \approx$ $1 \%, \gamma \approx 4 \%$ and $\gamma \approx 20 \%$ flows. Small difference may be attributed to the noise in the data. It may be noted that the eigenfunctions are normalized in the sense that $\left(\boldsymbol{\Phi}^{k}, \boldsymbol{\Phi}^{l}\right)=\delta_{k l}$, where $\delta_{k l}$ denotes the Kronecker delta. Similar Reynolds number invariance was also observed by Liu, Adrian \& Hanratty (1994) in a turbulent channel flow. Figure 20 also shows mode crossing in the sense that the fourth mode in the pre-transitional flow is similar to the third mode of the $\gamma \approx 20 \%$ flow. Mode crossing may occur due to slight change of flow parameters such as the Reynolds number, and hence change in the energy of the modes (e.g. Prabhu, Collis \& Chang 2001; Gunes \& Rist 2004).

The near-degenerate eigenspectrum of the POD modes (mentioned above) results from the symmetry in the flow (Aubry, Guyonnet \& Lima 1992; Rempfer \& Fasel 1994; Hasan \& Sanghi 2007). For clarity, only the $u$ component of the eigenfunctions corresponding to the degenerate eigenvalues in the $\gamma \approx 20 \%$ flow (grid 3 ) is shown in figure 23. The eigenfunctions appear nearly the same but shifted in space (figure 23c). In their POD study of the Tollmien-Schilichting wave-driven transitional flow in a flat-plate boundary layer, Rempfer \& Fasel (1994) also observed similar symmetry in the degenerate eigenfunctions. Aubry et al. (1992) found that the spacetime symmetry in the presence of travelling waves renders the POD eigenproblem degenerate. Also, the inviscid analysis of Andersson et al. (2001) shows that beyond a critical amplitude of streaks in the boundary layer, travelling waves are excited. Therefore, the symmetry inferred here may be a manifestation of some travelling wave in the streaky boundary layer; the deviation from exact symmetry in space and apparently large wavelength may be because of the fact that the wave may be a modulated one (e.g. Hasan \& Sanghi 2007) or just a component of a complete structure. 

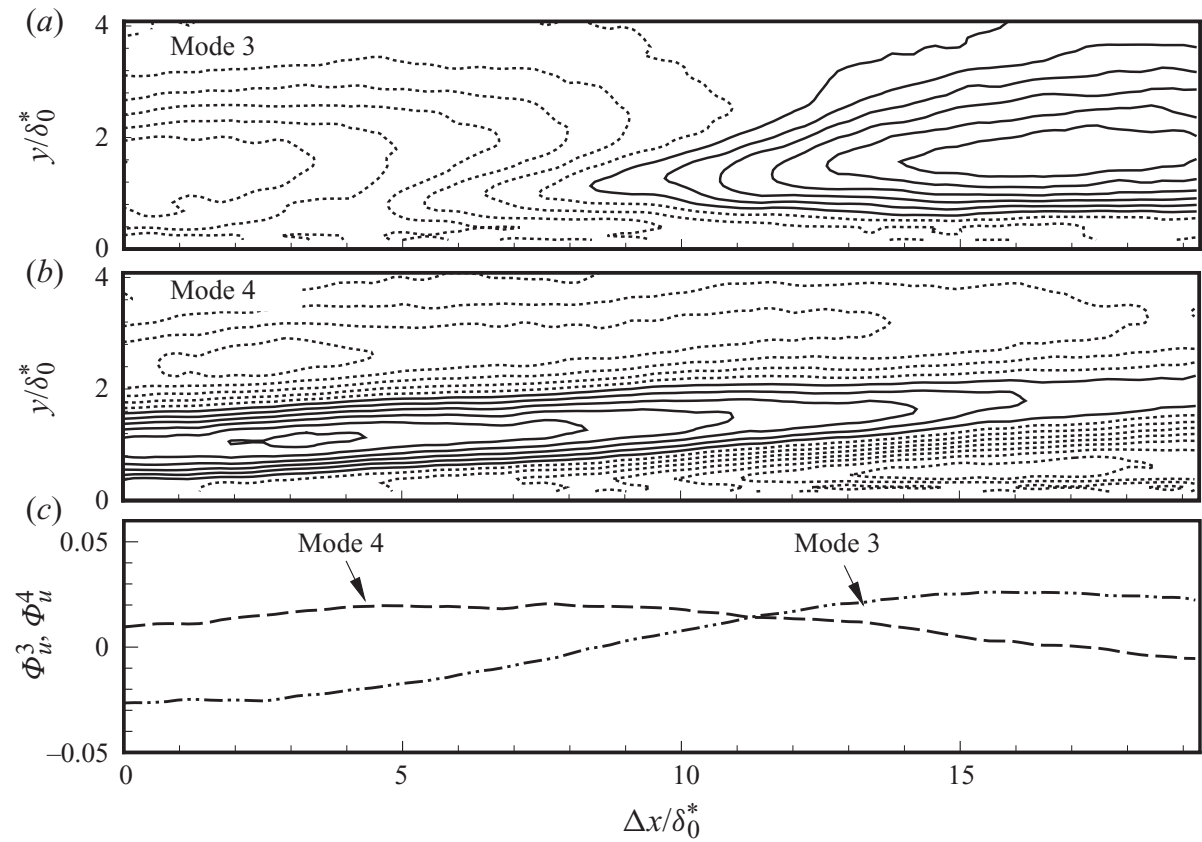

FiguRE 23. $(a, b)$ Streamwise component, $\Phi_{u}$, corresponding to the third and fourth eigenfunctions in the $\gamma \approx 20 \%$ flow for grid 3. Solid line, positive $\Phi_{u}$; dotted line, negative $\Phi_{u}$; contour level varies from 0.0285 to -0.027 with spacing 0.0043 . (c) Line plot of $\Phi_{u}^{3} \& \Phi_{u}^{4}$ at $y / \delta_{0}^{*} \approx 1.3$.

\section{Discussion}

Various experimental and numerical studies of bypass transition have brought out many important aspects of the streak breakdown process. However, a general consensus on the flow breakdown process is yet to be arrived at. Broadly, there have been two suggestions on the instability mechanism of streak for its eventual breakdown to turbulence. Several studies (e.g. Jacobs \& Durbin 2001; Zaki \& Durbin 2005; Durbin \& Wu 2007) suggest that the breakdown occurs due to the interaction of the lifted-up backward jets of the streamwise fluctuating velocity with free-stream eddies leading to the Kelvin-Helmholtz-type instability, while others (e.g. Brandt et al. 2004; Mans et al. 2007; Schlatter et al. 2008) propose that it is due to the secondary instability. However, the recent simulation by Schlatter et al. (2008) suggests these two mechanisms to be the same, when a streak undergoing secondary instability is observed simultaneously in both the planes.

This study shows that the lifted-up negative $u$ fluctuations in the wall-normal plane extend to the boundary-layer edge, in agreement with the numerical simulation of Jacobs \& Durbin (2001). These inclined negative $u$ fluctuations appear with the instantaneous inflectional velocity profiles, along with an inclined shear layer. Similar inflectional velocity profiles are also seen in the ribbon-induced transition experiment of Klebanoff et al. (1962). In fact, the inflectional velocity profile data at $\Delta x / \delta_{0}^{*} \approx 2$ in figures $11(b)$ and 12(a) collapse with those of Klebanoff et al. (1962), as shown in figure 24. Also, the instantaneous iso-shear lines in figure 12 and the oscillating inclined shear layers in figure 13 resemble the kink-like inclined shear layers in the controlled secondary instability studies in Poiseuille and boundary-layer flows (e.g. Nishioka et al. 1981; Rist \& Fasel 1995). The inclined shear with inflectional velocity 


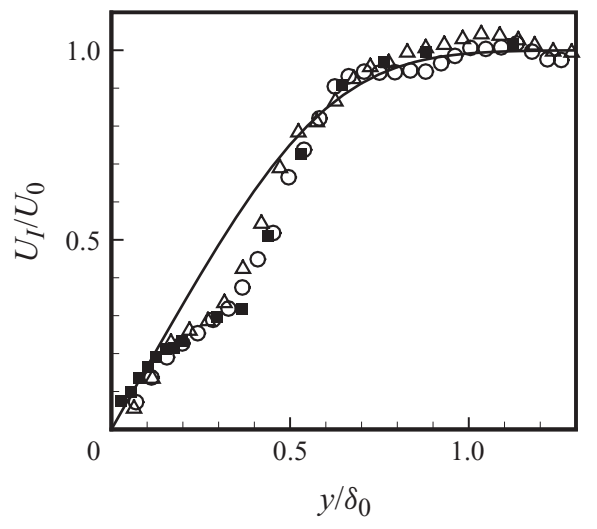

FIGURE 24. Comparison of the instantaneous velocity profiles at $\Delta x / \delta_{0}^{*} \approx 2$ locations in figures $11(b)$ and $12(a)$ with the instantaneous profile of Klebanoff et al. (1962). Symbols: $\triangle$, grid $1, \gamma \approx 1 \% ; O$, grid $3, \gamma \approx 1 \% ; \mathbf{\square}$, Klebanoff et al. (1962).

profile is an outcome of the secondary instability and it is the precursor of the flow breakdown (Klebanoff et al. 1962; Nishioka et al. 1981).

Although this study does not explicitly identify the role of varicose or sinuous mode in the breakdown process, an indication of their role in the breakdown process emerges. A varicose-like breakdown is associated with the wall-normal shear (Brandt et al. 2004) and the present breakdown scenario in the wall-normal plane appears to have originated from an oscillating wall-normal high shear layer. The wavelength in the case of sinuous breakdown of streaks is not constant and can vary from 7 to $20 \delta^{*}$ (Schlatter et al. 2008). This study also reveals a similar variation of wavelength (see figures 16 and 17). Also, the POD analysis of the PIV data reveals the possibility of the existence of travelling waves; this supports the results of the inviscid analysis of Andersson et al. (2001), who found that travelling waves are excited when streak amplitude is above $26 \%$ of the free-stream velocity.

\section{Summary}

Experimental studies of bypass transition in a flat-plate boundary layer have been carried out using the PIV technique. Due to the streak unsteadiness, the positive and negative fluctuations of the fluctuating streamwise velocity component, $u$, are found to dominate the flow. These positive and negative $u$ fluctuations resemble the jet-like structures seen in the simulation of bypass transition by Jacobs \& Durbin (2001), and therefore provide an experimental verification of the simulated picture. By conditional sampling of the fluctuating streamwise velocity component from the PIV realizations, these positive and negative fluctuations have been separated. The r.m.s. maxima of the positive fluctuations, $u_{r m s, f}$, and the negative fluctuations, $u_{r m s, b}$, are found to be of the same order; the peak $u_{r m s, f}$ is near the wall, whereas the peak $u_{r m s, b}$ occurs towards the edge of the boundary layer. Also, both $u_{r m s, f}$ and $u_{r m s, b}$ follow the non-modal growth, and the maximum amplitude in both these cases exceeds $25 \%$ of the free-stream speed in $\gamma \approx 1 \%$ flows. Importantly, the total streamwise fluctuating energy is found to be a linear combination of the positive and negative $u$ fluctuations, i.e. $u_{r m s}^{2} \approx 2 a^{2}\left(u_{r m s, f}^{2}+u_{r m s, b}^{2}\right), a=0.34$.

Instantaneous inflectional velocity profiles are found to emerge along with an inclined shear layer and a structure of the negative streamwise fluctuating velocity 
component in transitional flows. This instantaneous inflectional velocity profile is remarkably similar to that in the ribbon-induced flow breakdown studied by Klebanoff et al. (1962). Although the Tollmien-Schlichting wave mechanism for the initial growth of disturbance is absent in bypass transition, an important conclusion here is that the breakdown stage involving the instantaneous inflectional velocity profile seems similar in ribbon-induced and bypass transitions.

The lifted-up inclined shear layer and its oscillation (figure 13a) appear to be the turbulent spot precursor. A turbulent spot identified from the PIV realizations provided an opportunity to compare it with artificial turbulent spots studied in the past (e.g. Wygnanski et al. 1982; Singer 1996). The turbulent spot so identified is found to contain the features of an artificial turbulent spot - a lobe of negative streamwise fluctuating velocity, $u$, seating on a lobe of positive $u$. To the best of our knowledge, this PIV picture of a spot possibly is the first one of an actual turbulent spot. The maximum velocity perturbation in the turbulent spot is found to be $\mp 30 \%$ of the free-stream speed, in agreement with that for an artificial turbulent spot (Wygnanski et al. 1982). Establishing all the characteristics of a turbulent spot in bypass transition remains a challenging task and further PIV studies are expected to provide more details.

The POD analysis of the two-dimensional PIV data in the wall-normal plane reveals that the main bypass transition scenario may be described by a few dominant eigenfunction modes. Moreover, the first two dominant eigenfunction modes show self-similarity for different Reynolds numbers and free-stream turbulence levels, which suggests that the flow prevails its structural identity in transitional flows. These aspects may be useful in designing a feedback control strategy for the control of bypass transition. The degenerate POD modes suggesting the possible existence of the travelling waves in the flow are also found to be related to the inclined shear layers. Therefore, it is suggested that an inclined shear layer at the breakdown stage of bypass transition is associated with a propagating structure.

We sincerely thank the Department of Science and Technology for funding the PIV unit through the FIST programme. The financial support from AR and DB through a project is gratefully acknowledged. We would also like to thank the referees for their fruitful suggestions and comments which improved the paper.

\section{REFERENCES}

Adrian, R. J., Christensen, K. T. \& LiU, Z. C. $2000 a$ Analysis and interpretation of instantaneous turbulent velocity fields. Exp. Fluid 29, 275-290.

Adrian, R. J., Meinhart, C. D. \& Tomkins, C. D. $2000 b$ Vortex organization in the outer region of the turbulent boundary layer. J. Fluid Mech. 422, 1-54.

Alfredsson, P. H. \& Matsubara, M. 2000 Free-stream turbulence, streaky structures and transition in boundary layer flows. AIAA Paper 2000-2534.

Andersson, P., Berggren, M. \& Henningson, D. S. 1999 Optimal disturbances and bypass transition in boundary layers. Phys. Fluids. 11, 134-150.

Andersson, P., Brandt, L., Bottaro, A. \& Henningson, D. 2001 On the breakdown of boundary layer streaks. J. Fluid Mech. 428, 29-60.

Arnal, D. \& Juillen, J. C. 1978 Contribution experimentale à l'ètude de la receptivité d'une couche limite laminarire, $\grave{a}$ la turbulence de l'ecoulement general. Rap. Tech. 1/5018 AYD ONERA.

Asai, M., Minagawa, M. \& Nishioka, M. 2002 The instability and breakdown of a near-wall low-speed streak. J. Fluid Mech. 455, 289-314.

Aubry, N., Guyonnet, R. \& Lima, R. 1992 Spatio-temporal symmetries and bifurcations via bi-orthogonal decompositions. J. Nonlinear Sci. 2, 183-215. 
Aubry, N., Holmes, P., Lumley, J. L. \& Stone, E. 1988 The dynamics of coherent structures in the wall region of a turbulent boundary layer. J. Fluid Mech. 192, 115-173.

Banerjee, A. S., Mandal, A. C. \& Dey, J. 2006 Particle image velocimetry studies of an incipient spot in the Blasius boundary layer. Exp. Fluids 40, 928-941.

Berkooz, G., Holmes, P. \& Lumley, J. L. 1993 The proper orthogonal decomposition in the analysis of turbulent flows. Annu. Rev. Fluid Mech. 25, 539-575.

BLAIR, M. F. 1992 Boundary layer transition in accelerating flows with intense freestream turbulence: Part 1. Disturbances upstream of transition onset. Trans. ASME: J. Fluid Engng 144, 313-321.

BRANDT, L. \& Henningson, D. S. 2002 Transition of streamwise streaks in zero-pressure-gradient boundary layers. J. Fluid Mech. 472, 229-261.

Brandt, L., Schlatter, P. \& Henningson, D. S. 2004 Transition in boundary layers subject to free-stream turbulence. J. Fluid Mech. 517, 167-198.

Butler, K. M. \& Farrell, B. F. 1992 Three-dimensional optimal perturbations in viscous shear flow. Phys. Fluids A 4, 1637-1650.

Cazemier, W., Verstappen, R. W. C. P. \& Veldman, A. E. P. 1998 Proper orthogonal decomposition and low-dimensional models for driven cavity flows. Phys. Fluids 10, 1685-1699.

Chong, M. S., Perry, A. E. \& Cantwell, B. J. 1990 A general classification of three-dimensional flow fields. Phys. Fluids 2, 765-777.

Deane, E., Kevrekidis, I. G., Karniadakis, G. E. \& Orszag, S. A. 1991 Low-dimensional models for complex geometry flows: application to grooved channels and circular cylinders. Phys. Fluids 3, 2337-2354.

Dryden, H. L. 1936 Air flow in the boundary layer near a plate. Tech. Rep. 562. NASA.

Durbin, P. \& Wu, X. 2007 Transition beneath vortical disturbances. Annu. Rev. Fluid Mech. 39, $107-128$.

Elofsson, P. A., Kawakami, M. \& Alfredsson, P. H. 1999 Experiments on the stability of streamwise streaks in plane Poiseuille flow. Phys. Fluids 11, 915-930.

Fransson, J. H. M., Matsubara, M. \& Alfredsson, P. H. 2005 Transition induced by free-stream turbulence. J. Fluid Mech. 527, 1-25.

Gunes, H., Liakopoulos, A. \& Sahan, R. A. 1997 Low-dimensional description of oscillatory thermal convection: the small Prandtl number limit. Theor. Comput. Fluid Dyn. 9, 1-16.

Gunes, H. \& Rist, U. 2004 Proper orthogonal decomposition reconstruction of a transitional boundary layer with and without control. Phys. Fluids 16, 2763-2784.

Hasan, N. \& SANGHI, S. 2007 Proper orthogonal decomposition and low-dimensional modelling of thermally driven two-dimensional flow in a horizontal rotating cylinder. J. Fluid Mech. 573, 265-295.

Hernon, D., Walsh, E. J. \& Mceligot 2007 Experimental investigation into the routes to bypass transition and the shear-sheltering phenomenon. J. Fluid Mech. 591, 461-479.

Holmes, P., Lumley, J. L. \& Berkooz, G. 1996 Turbulence, Coherent Structures, Dynamical Systems and Symmetry. Cambridge University Press.

Inasawa, A., Lundell, F., Matsubara, M., Kohama, Y. \& Alfredsson, P. H. 2003 Velocity statistics and flow structures observed in bypass transition using stereo PTV. Exp. Fluids 34, 242252.

Jacobs, R. G. \& Durbin, P. A. 2001 Simulations of bypass transition. J. Fluid Mech. 428, 185-212.

Kendall, J. M. 1985 Experimental study of disturbances produced in a pre-transitional laminar boundary layer by weak free stream turbulence. AIAA Paper 85-1695.

Kendall, J. M. 1990 Boundary layer receptivity to free-stream turbulence. AIAA Paper 90-1504.

Kendall, J. M. 1991 Studies on laminar boundary layer receptivity to free stream turbulence near a leading edge. In Boundary Layer Stability and Transition to Turbulence (ed. D. C. Reda, H. L. Reed \& R. Kobayashi) vol. 114, pp. 23-30. ASME-FED.

Kendall, J. M. 1998 Experiments on boundary layer receptivity to freestream turbulence. AIAA Paper 98-0530.

KLEBANOFF, P. S. 1971 Effect of freestream turbulence on the laminar boundary layer. Bull. Am. Phys. Soc. 10, 1323.

Klebanoff, P. S., Tidstrom, K. D. \& Sargent, L. M. 1962 The three dimensional nature of boundary-layer instability. J. Fluid Mech. 12, 1-34.

Kostas, J., Soria, J. \& Chong, M. S. 2002 Particle image velocimetry measurements of a backwardfacing step flow. Exp. Fluids 33, 838-853. 
Krothapalli, A., Venkatakrishnan, L., Lourenco, L., Greska, B. \& Elavarasan, R. 2003 Turbulence and noise suppression of a high-speed jet by water injection. J. Fluid Mech. 491, 131-159.

Kruse, N., Gunther, A. \& Rohr, P. R. V. 2003 Dynamics of large-scale structures in turbulent flow over wavy wall. J. Fluid Mech. 485, 87-96.

Leventhal, L. \& Reshotкo, E. 1981 Preliminary experimental study of disturbances in a laminar boundary layer due to free stream turbulence. AIAA Paper 81-1224.

LiU, Z., Adrian, R. J. \& HanRatty, T. J. 2001 Large-scale modes of turbulent channels flow: transport and structure. J. Fluid Mech. 448, 53-80.

Liu, Z.-C., Adrian, R. J. \& Hanratty, T. J. 1994 Large-scale modes of turbulent channels flow: transport and structure. Phys. Fluids 6, 2815-2819.

Lourenco, L. M. \& Krothapalli, A. 2000 TRUE resolution PIV: a mesh-free second order accurate algorithm. In Proceedings of the International Conference in Applications of Lasers to Fluid Mechanics, Lisbon, Portugal.

LUCHINI, P. 2000 Reynolds-number-independent instability of the boundary layer over a flat surface: optimal perturbations. J. Fluid Mech. 404, 289-309.

Lumley, J. L. 1967 The structure of inhomogeneous turbulence. In Atmospheric Turbulence and Wave Propagation (ed. A. M. Yaglom \& V. I. Tatarski), pp. 166-178. Nauka.

Mandal, A. C. 2005 Particle image velocimetry (PIV) measurements in a low intermittency transitional flow. Master's thesis, Indian Institute of Science, Bangalore, India.

Mandal, A. C., Venkatakrishnan, L. \& Dey, J. 2006 Spot-like structure in free-stream induced transitional flow. In 6th European Fluid Mechanics Conference, Stockholm, Sweden.

Mans, J., Kadijk, E. C., De Lange, H. C. \& van Steenhoven, A. A. 2005 Breakdown in a boundary layer exposed to free-stream turbulence. Exp. Fluids 39, 1071-1083.

Mans, J., De Lange, H. C. \& van Steenhoven, A. A. 2007 Sinuous breakdown in a flat plate boundary layer exposed to free-stream turbulence. Phys. Fluids 19, 088101-1-088101-4.

Matsubara, M. \& Alfredsson, P. H. 2001 Disturbance growth in boundary layers subjected to free-stream turbulence. J. Fluid Mech. 430, 149-169.

Moreno, D., Krothapalli, A., Alkislar, M. B. \& Lourenco, L. M. 2004 Low-dimensional model of a supersonic rectangular jet. Phys. Rev. E 69, 026304-1-026304-12.

Morkovin, M. V. 1969 The many faces of transition. In Viscous Drag Reduction (ed. C. S. Wells), pp. 1-31. Plenum Press.

Narasimha, R., Devasia, K. J., Gururani, G. \& Narayanan, M. A. Badri 1984 Transitional intermittency in boundary layers subjected to pressure gradient. Exp. Fluids 2, 171-176.

Narasimha, R. \& Prasad, S. N. 1994 Leading edge shape for flat plate boundary layer studies. Exp. Fluids 17, 358-360.

Nishioka, M., AsaI, M. \& IIDA, S. 1981 Wall phenomena in the final stage of transition to turbulence. In Transition and Turbulence (ed. R. E. Meyer), pp. 113-126. Academic Press.

Pedersen, J. M. \& Meyer, K. E. 2002 POD analysis of flow structures in scale model of a ventilated room. Exps. Fluids 33, 940-949.

Perry, A. E. 1982 Hot-Wire Anemometry. Clarendon Press.

Prabhu, R. D., Collis, S. S. \& Chang, Y. 2001 The influence of control on proper orthogonal decomposition of wall-bounded turbulent flows. Phys. Fluids 13, 520-537.

Rajaee, M., Karlsson, S. K. F. \& Sirovich, L. 1994 Low-dimensional description of free-shear-flow coherent structures and their dynamical behaviour. J. Fluid Mech. 258, 1-29.

Ramesh, O. N., Dey, J. \& Prabhu, A. 1996 Transitional intermittency distribution in a threedimensional constant pressure diverging flow. Exp. Fluids 21, 259-263.

REMPFER, D. \& FASEL, H. F. 1994 Evolution of three-dimensional coherent structures in a flat-plate boundary layer. J. Fluid Mech. 260, 351-375.

Rist, U. \& FASEL, H. 1995 Direct numerical simulation of controlled transition in a flat-plate boundary layer. J. Fluid Mech. 298, 211-248.

RoACH, P. E. \& BRIERLY, D. H. 1992 The influence of a turbulent free stream on zero pressure gradient transitional boundary layer development. Part I. Test cases T3A and T3B. In ERCOFTAC Workshop: Numerical Simulation of Unsteady Flows and Transition to Turbulence, Lausanne (ed. O. Pironneau, W. Rodi, I. L. Ryhming, A. M. Savill \& T. V. Truong), pp. 319-347. Cambridge University Press. 
Schlatter, P., Brandt, L., De Lange, H. C. \& Henningson, D. S. 2008 On streak breakdown in bypass transition. Phys. Fluids 20, 101505-1-101505-15.

Simon, T. W., QIU, S. \& Yuan, K. 2000 Measurements in a transitional boundary layer under low-pressure turbine airfoil conditions. Tech. Rep. CR-2000-209957. NASA.

Singer, B. A. 1996 Characteristics of a young turbulent spot. Phys. Fluids 8, 509-521.

Sirovich, L. 1987 Turbulence and the dynamics of coherent structures. Part 1: Coherent structures. Q. Appl. Math. 45, 561-571.

TAYLOR, G. I. 1939 Some recent developments in the study of turbulence. In Proceedings of Fifth International Congress for Applied Mechanics (ed. J. P. Den Hartog \& H. Peters), pp. 294-310. Wiley.

Tennekes, H. \& Lumley, J. L. 1997 A First Course in Turbulence. MIT Press.

Vasudevan, K. P., Dey, J. \& Prabhu, A. 2001 Spot propagation characteristics in laterally strained boundary layer. Exp. Fluids 30, 488-491.

Westin, K. J. A., Boiko, A. V., Klingmann, B. G. G., Kozlov, V. V. \& Alfredsson, P. H. 1994 Experiments in a boundary layer subjected to free stream turbulence. Part 1. Boundary layer structure and receptivity. J. Fluid Mech. 281, 193-218.

Wygnanski, I., Zilberman, M. \& HaRitonidis, J. H. 1982 On the spreading of a turbulent spot in the absence of a pressure gradient. J. Fluid Mech. 123, 69-90.

ZAKI, T. A. \& Durbin, P. A. 2005 Mode interaction and the bypass route to transition. J. Fluid Mech. 531, 85-111. 